ARTICLE

Received 3 Oct 2015 | Accepted 9 Feb 2016 | Published 22 Mar $2016 \quad$ DOl: 10.1038/ncomms10993 OPEN

\title{
MicroRNA-378 limits activation of hepatic stellate cells and liver fibrosis by suppressing Gli3 expression
}

Jeongeun Hyun ${ }^{1}$, Sihyung Wang ${ }^{1}$, Jieun Kim¹, Kummara Madhusudana Rao ${ }^{2}$, Soo Yong Park ${ }^{2}$, Ildoo Chung², Chang-Sik $\mathrm{Ha}^{2}$, Sang-Woo Kim¹,3, Yang H. Yun ${ }^{4} \&$ Youngmi Jung ${ }^{1,3}$

Hedgehog $(\mathrm{Hh})$ signalling regulates hepatic fibrogenesis. MicroRNAs (miRNAs) mediate various cellular processes; however, their role in liver fibrosis is unclear. Here we investigate regulation of miRNAs in chronically damaged fibrotic liver. MiRNA profiling shows that expression of miR-378 family members (miR-378a-3p, miR-378b and miR-378d) declines in carbon tetrachloride $\left(\mathrm{CCl}_{4}\right)$-treated compared with corn-oil-treated mice. Overexpression of miR-378a-3p, directly targeting Gli3 in activated hepatic stellate cells (HSCs), reduces expression of Gli3 and profibrotic genes but induces gfap, the inactivation marker of HSCs, in $\mathrm{CCl}_{4}$-treated liver. Smo blocks transcriptional expression of miR-378a-3p by activating the p65 subunit of nuclear factor $-\kappa B(N F-\kappa B)$. The hepatic level of miR-378a-3p is inversely correlated with the expression of Gli3 in tumour and non-tumour tissues in human hepatocellular carcinoma. Our results demonstrate that miR-378a-3p suppresses activation of HSCs by targeting Gli3 and its expression is regulated by Smo-dependent NF- $\kappa$ B signalling, suggesting miR-378a-3p has therapeutic potential for liver fibrosis.

\footnotetext{
${ }^{1}$ Department of Integrated Biological Science, College of Natural Science, Pusan National University, Pusan 46241, Korea. ${ }^{2}$ Department of Polymer Science and Engineering, College of Engineering, Pusan National University, Pusan, 46241, Korea. ${ }^{3}$ Department of Biological Sciences, College of Natural Science, Pusan National University, 63-2 Pusandaehak-ro, Kumjeong-gu, Pusan 46241, Korea. ${ }^{4}$ Department of Biomedical Engineering, College of Engineering, The University of Akron, Akron, Ohio 44685-0302, USA. Correspondence and requests for materials should be addressed to Y.J. (email: y.jung@pusan.ac.kr).
} 
C hronic liver diseases such as cirrhosis and liver cancer are a leading cause of death worldwide ${ }^{1}$. Liver fibrosis, a major characteristic of most chronic liver diseases, accelerates the progression of liver disease to the chronic form by destroying the normal hepatic parenchyma ${ }^{2}$. Therefore, understanding fibrogenesis is important for the development of effective antifibrotic therapies.

MicroRNAs (miRNAs) are endogenous non-coding RNAs of $\sim 22$ nucleotides in length, which are conserved across species ${ }^{3}$. They recognize their target genes through base pairing between a sequence of around six to eight nucleotides in length at their $5^{\prime}$-end (called the seed sequence) and the complementary sequence within the messenger RNA of target genes, suppressing the expression of these target genes at the posttranscriptional level by mRNA decay or by translational inhibition via an miRNA-containing RNA-induced silencing complex ${ }^{4}$. Mammalian miRNAs have been implicated in various biological processes, such as differentiation, proliferation, oxidative stress resistance and tumour suppression ${ }^{5,6}$. In addition, miRNAs are known to be dysregulated in several diseases, including cancers. Because of their gene-regulating ability, miRNAs are attractive therapeutic targets. However, most research has focused on the functions of miRNAs in cancer and little is known about the mechanisms of how miRNAs are involved in hepatic pathological processes, including fibrosis.

The Hedgehog (Hh) signalling pathway promotes liver fibrosis $^{7,8}$. The Hh ligands, Sonic Hh, Indian $\mathrm{Hh}$ and Desert $\mathrm{Hh}$, bind to the $\mathrm{Hh}$ receptor Patched (Ptc) and release Smoothened (Smo) into the cytoplasm. Cytoplasmic Smo translocates the Glioblastoma (Gli) family proteins (including Gli1, Gli2 and Gli3) into the nucleus, where they act as transcriptional activators of $\mathrm{Hh}$ signalling ${ }^{8}$. In the damaged liver, hepatocytes undergoing apoptosis secrete Hh ligands that initiate proliferation of hepatic progenitor cells and hepatic stellate cells (HSCs) ${ }^{9,10}$. These cells in turn produce $\mathrm{Hh}$ ligands and further accelerate Hh signalling in an autocrine and paracrine manner. During this process, Hh signalling promotes activation of quiescent HSCs into myofibroblastic HSCs, which contribute to the accumulation of fibrous extracellular matrix (ECM) in the liver ${ }^{11,12}$. Hence, Glis are good targets for biomarker and therapeutic approaches aimed at controlling activation of HSCs during liver fibrogenesis.

Here we investigate the involvement of miRNAs in the progression of liver fibrosis, with miRNA array analysis of chronically damaged and fibrotic mouse livers. MiR-378a-3p and its family members, miR-378b and miR-378d, are the most significantly downregulated miRNAs in carbon tetrachloride $\left(\mathrm{CCl}_{4}\right)$-treated livers compared with corn-oil-treated (control) livers. The miR-378 family is downregulated during HSC activation and suppresses activation of HSCs by targeting gli3. In addition, the effect of miR-378a-3p on gli3 is mediated by transcriptional regulation of the nuclear factor- $\kappa \mathrm{B}(\mathrm{NF}-\kappa \mathrm{B})$ p65 subunit, interacting with Smo. To explore the clinical implications, we encapsulate miR-378a-3p with nanoparticles (NPs) fabricated with L-tyrosine polyurethane (LTU2a), a biodegradable polymer composed of a modified amino acid, PEG and hexamethylene diisocynate in its backbone ${ }^{13,14}$. In-vivo application reduces the expression of Gli3 and matrix markers, and leads to the recovery of fibrosis. These results suggest that miR-378 targeting $\mathrm{Hh}$ signalling is a novel diagnostic and therapeutic target for understanding and treating the pathogenesis of chronic liver disease.

\section{Results}

MiR-378 is repressed by $\mathrm{CCl}_{4}$-induced chronic liver injury. $\mathrm{CCl}_{4}$ is a hepatotoxic chemical that effectively induces liver fibrosis in experimental rodent models ${ }^{15}$. To generate the experimental model of hepatic fibrosis, we exposed mice to $\mathrm{CCl}_{4}$ twice a week for $6(n=5)$ and $10(n=6)$ weeks by intraperitoneal (i.p.) injection. As a control group for comparison, an equal number of mice were treated with corn oil. Compared with cornoil treatment, $\mathrm{CCl}_{4}$ treatment increased the ratio of liver to body weight in mice (Supplementary Fig. 1a). Haematoxylin and eosin $(\mathrm{H} \& \mathrm{E})$ staining showed that in liver from $\mathrm{CCl}_{4}$-injected mice, there were increases in centrilobular necrosis and the number of apoptotic hepatocytes, and that a large number of immune cells had infiltrated into the hepatic parenchyma (Supplementary Fig. 1b). The RNA level of $\alpha$-smooth muscle actin $(\alpha-s m a)$ and collagen type $1-\alpha 1(\operatorname{col} 1 \alpha 1)$, and the protein level of Tgf- $\beta$ and $\alpha$ SMA were higher in livers of $\mathrm{CCl}_{4}$-treated mice than in the liver of control mice (corn-oil-treated mice) (Supplementary Fig. 2a-c). Sirius red stain revealed deposition of excessive collagen fibres in $\mathrm{CCl}_{4}$-treated livers and the hydroxyproline content was higher in livers of $\mathrm{CCl}_{4}$-treated mice than in the liver of own control mice (Supplementary Fig. 2d,e). In addition, immunostaining for $\alpha$ SMA, an activated HSC (aHSC) marker ${ }^{16}$, showed that myofibroblastic HSCs had proliferated along the fibrotic tract (Supplementary Fig. 2f). These results confirmed that $\mathrm{CCl}_{4}$ induces severe hepatic fibrosis in mice.

To investigate the differences in miRNA expression profiles between fibrotic and normal livers, we performed miRNA microarrays for total RNA extracts isolated from mouse livers treated with $\mathrm{CCl}_{4}$ or corn oil for 10 weeks ( $n=3$ per group). MiRNAs were considered to have significant differences in expression level when the expression difference showed more than twofold change between the experimental and control groups at $P<0.05$. We found that 12 miRNAs were differentially expressed in fibrotic liver. Seven miRNAs (mmu-miR-574-5p, mmu-miR-466i-5p, mmu-miR-342-3p, mmu-let7i-5p, mmumiR-34a-5p, mmu-miR-188-5p and mmu-miR-5119) were upregulated and the other five (mmu-miR-378a-3p, mmu-miR202-3p, mmu-miR-378b, mmu-miR-378d and mmu-miR-212$3 \mathrm{p})$ were downregulated in the $\mathrm{CCl}_{4}$ group compared with the control (Fig. 1a,b). Among these miRNAs that were dysregulated in liver fibrosis, several miR-378 family members, including miR378a-3p (0.395-fold), miR-378b (0.390-fold) and miR-378d (0.372-fold), had the lowest expression in the livers of $\mathrm{CCl}_{4}$ treated mice. This reduced expression of miR-378 family members was validated by real-time quantitative reverse transcriptase-PCR (qRT-PCR) analysis, which showed that miR-378a-3p was significantly decreased, and miR-378b and miR-378d tended to be reduced in fibrotic livers compared with controls ( $n=4$ per group) both at 6 and 10 weeks (unpaired twosample Student's $t$-test, $P<0.05$ ) (Fig. 1c). In addition, decreased expression of $\mathrm{miR}-378 \mathrm{a}-3 \mathrm{p}$ in these mice was significantly correlated with the degree of liver fibrosis, as assessed by hydroxyproline assay (Spearman's rank correlation analysis, $r=-0.475, P=0.034, n=20$ ) (Fig. 1d).

Microarray and qRT-PCR analyses showed that the expressional level of miR-378a-5p was not significantly changed in fibrotic liver, although it was transcribed together with miR-378 a-3p (Supplementary Fig. 3). MiR-378a-5p was upregulated and influenced SULT2A1 expression in primary sclerosing cholangitis $(\mathrm{PSC})^{17}$. Wunsch et al. ${ }^{17}$ demonstrated that PSC is characterized by disease-specific impairment of SULT2A1 expression, which is regulated by miR-378a-5p. These findings suggest that expressional pattern of miR-378a-5p seems to be PSC specific. In line with their findings, miR-378a-3p, not the $5 \mathrm{p}$, shows the significant changes in $\mathrm{CCl}_{4}$-treated mice and the association with HSC activation. These findings suggest the possibility that miR-378a-3p and miR-378a-5p have disease-specific effects in liver. Hence, further study is needed to investigate the potential role of miR-378 in PSC. 
a

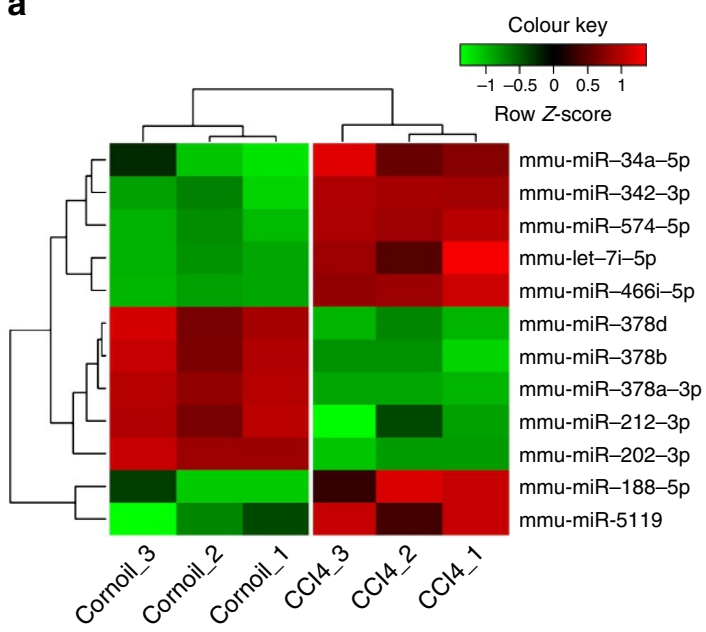

b

\begin{tabular}{lcr}
\hline \multicolumn{3}{c}{ Downregulated miRNAs } \\
\hline \multicolumn{1}{c}{ miRNA } & $\begin{array}{l}\text { Fold change } \\
\left(\mathrm{CCl}_{4} / \mathrm{CON}\right)\end{array}$ & Adj. $P$ - value \\
\hline miR-378a-3p & 0.39536 & 0.00162 \\
miR-378b & 0.38952 & 0.00327 \\
miR-378d & 0.37246 & 0.00327 \\
miR-202-3p & 0.47332 & 0.00327 \\
miR-212-3p & 0.45606 & 0.01896 \\
\hline \multicolumn{3}{c}{ Upregulated miRNAs } \\
\hline miRNA & Fold change $^{\left(C_{4}\right.}$ Adj. $P$ - value \\
\hline miR-574-5p & 12.33949 \\
miR-466i-5p & 8.48176 & 0.00026 \\
miR-342-3p & 2.26664 & 0.00083 \\
Let-7i-5p & 2.19846 & 0.00327 \\
miR-34a-5p & 3.65902 & 0.01625 \\
miR-188-5p & 6.50153 & 0.03288 \\
miR-5119 & 2.06774 & 0.04278 \\
\hline
\end{tabular}

C
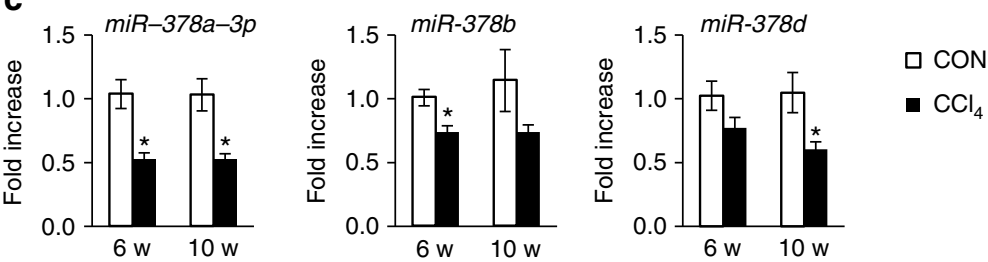

d

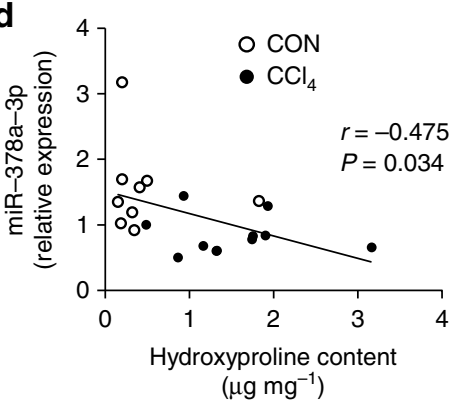

Figure 1 | MiR-378 family was downregulated in injured livers of $\mathbf{C C l}_{\mathbf{4}}$-treated mice. (a) Microarray analysis for miRNA expression was performed with total RNA extracted from livers of mice treated with corn-oil (control; $\mathrm{CON}$ ) or $\mathrm{CCl}_{4}$ ( $n=3$ per group) for 10 weeks. Heat map shows the two-way hierarchical clustering of differentially expressed miRNAs. Each row and column represents an miRNA and a condition, respectively. The row Z-score scaling for the expression level of each miRNA was calculated by subtracting the mean expression of the miRNA from its expression value and then dividing by the s.d. across all the samples. The closer the colour is to bright green, the lower the expression; the closer to bright red, the higher the expression. (b) A list of significantly dysregulated miRNAs in $\mathrm{CCl}_{4}$-treated compared with corn-oil-treated livers (CON) is shown, with the fold change and $P$-values (c) qRT-PCR was performed to assess expression of the miR-378 family, including miR-378a-3p, miR-378b and miR-378d, in livers from the CON-and $\mathrm{CCl}_{4}$-treated mice at 6 and 10 weeks ( $n=4$ per group). Mean \pm s.e.m. results are graphed (unpaired two-sample Student's $t$-test, ${ }^{\star} P<0.05$ versus $C O N$ ). (d) Spearman's rank correlation between miR-378a-3p expression and hydroxyproline contents in liver of all mice $(n=20$, Spearman's rank correlation analysis; $r$, correlation coefficient).

Expression of miR-378 is reduced during HSC activation. As the aHSC is a major ECM-producing cell contributing to liver fibrosis $^{18,19}$, we examined the expression of miR-378 family members in LX2, a human aHSC line. Expression of all of three miR-378 family members was reduced to a greater extent in LX2 than in HepG2, a human hepatocyte cell line (Fig. 2a). As primary HSCs are known to be activated during culture ${ }^{20,21}$, we isolated primary HSCs from mice and assessed whether the expression level of these miRNAs was changed during HSC culture. We found that miR-378a-3p, miR-378b and miR-378d were significantly downregulated at day 7 compared with day 0 of culture (Fig. 2b). To confirm these findings in vivo, we isolated primary $\mathrm{HSCs}$ from corn-oil- or $\mathrm{CCl}_{4}$-treated mice (Supplementary Figs 1 and 2). The expression of miR-378a-3p, miR-378b and miR-378d was also lower in primary aHSCs isolated from $\mathrm{CCl}_{4}$-treated mice than inactivated cells from cornoil-treated mice (Fig. 2c). Those results indicate that these miRNAs were downregulated during HSC activation. In addition, we examined the expression of miR-378 family members in different types of liver cells, including hepatocytes, quiescent (day 0) HSCs (qHSCs) and liver sinusoidal endothelial cells (LSECs), primarily isolated from livers of normal mice. Quiescent HSCs contained a higher level of miR-378a-3p and similar levels of miR-378b and miR-378d compared with hepatocytes. Expression 
a $\quad m i R-378 a-3 p$

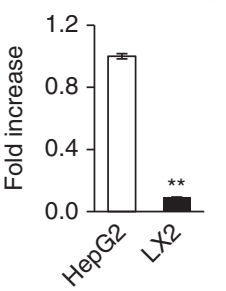

b $\quad m i R-378 a-3 p$

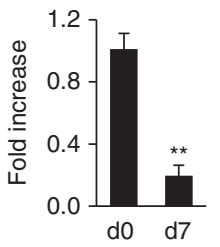

C $\quad m i R-378 a-3 p$

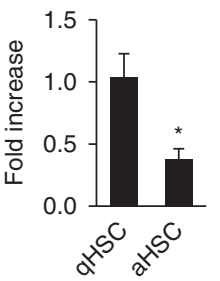

d

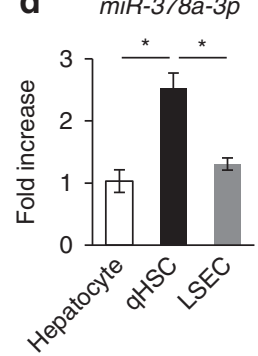

$m i R-378 b$

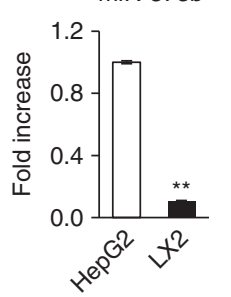

miR-378b

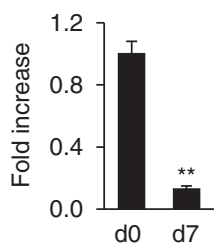

$\operatorname{miR}-378 b$

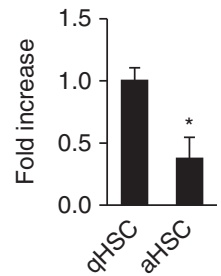

$\operatorname{miR}-378 b$

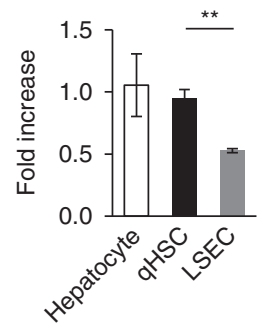

miR-378d

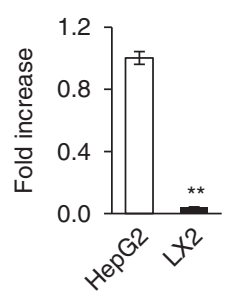

miR-378d

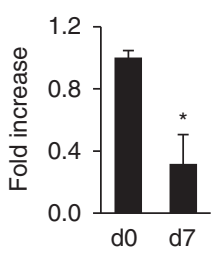

miR-378d

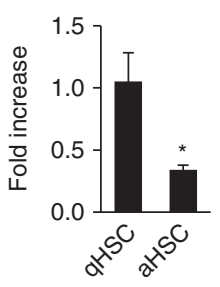

miR-378d

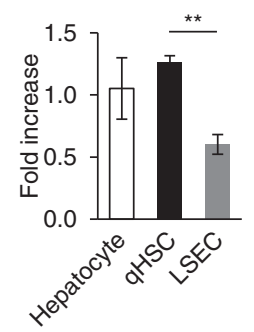

Figure 2 | Decreased expression of the miR-378 family in aHSCs.

(a) Expression of miR-378 family members, including miR-378a-3p, miR-378b and miR-378d, in HepG2 (human liver epithelial cell line) and LX2 (human aHSC line) cells was examined by qRT-PCR. (b) qRT-PCR of the miR-378 family in primary qHSCs isolated from normal C57BL/6 mice at quiescent stage ( $\mathrm{dO}$; immediately after isolation) and in primary aHSCs (d7; cultured for 7 days). (c) qRT-PCR of the miR-378 family in primary $\mathrm{HSCs}$ isolated from corn-oil- ( $\mathrm{qHSCs}$ ) or $\mathrm{CCl}_{4}$-(aHSCs)-treated mice.

(d) Expression of the miR-378 family in primary hepatocytes, primary qHSC and LSECs isolated from normal C57BL/6 mice, as assessed by QRT-PCR. All results of relative expression values are shown as mean \pm s.e.m. of triplicate experiments (unpaired two-sample Student's t-test, ${ }^{\star} P<0.05$ and $\left.{ }^{\star \star} P<0.005\right)$

of all three miR-378 family member was greater in qHSCs than in primary LSECs (Fig. 2d). These results suggested that the miR-378 family is involved in HSC activation.

MiR-378a-3p directly binds to Gli3 mRNA. To identify the relevant target genes of the miR-378 family, we conducted bioinformatic analysis using http://www.microrna.org/, a comprehensive resource of miRNA target predictions ${ }^{22}$. We found that gli2 and gli3, downstream transcriptional factors of $\mathrm{Hh}$ signalling, were potential targets of miR-378a-3p, both in human and mouse, two species that share common miR-378a-3p seed

sequences (Fig. 3a). Hence, we investigated whether miR-378a-3p interacted with the binding sites at the $3^{\prime}$-untranslated region ( $3^{\prime}$-UTR) of gli2 and gli3 mRNAs (Fig. 3b) using luciferase assay in neuroblasoma cell line $(\mathrm{N} 2 \mathrm{a})$. To fit the species of the experimental animals, we employed this cell line originated from mouse. Luciferase reporter assay revealed that miR-378a-3p, not miR-378b or miR-378d, directly bound to gli3 but not gli2 mRNA, and that both of the two binding sites of the gli3 mRNA were active (Fig. 3c and Supplementary Fig. 4). Expression of Gli3 and Gli2 at both the RNA and protein levels was elevated, whereas expression of gli1 was reduced in livers of $\mathrm{CCl}_{4^{-}}$ treated mice (Supplementary Fig. 5a-c). Pritchett et al. ${ }^{23}$ also demonstrated that gli1 is sparse in HSCs and does not increase on activation of HSCs. Interestingly, the amount of miR-378a-3p expression was inversely correlated with the hepatic level of Gli3 expression in those mice (Spearman's rank correlation analysis, $r=-0.733, P=0.007, n=12$ ) (Supplementary Fig. 5d). In addition, LX2 cells showed a larger increase in gli3 and gli2, compared with HepG2 cells (Supplementary Fig. 5e). These results suggest that upregulation of Gli3 expression is related to significant reduction of miR-378a-3p targeting gli3 in chronically damaged liver.

MiR-378a-3p suppresses activation of HSCs. We investigated whether ectopic expression of miR-378a-3p in the aHSCs influenced HSC activation. Therefore, we isolated primary aHSCs from $\mathrm{CCl}_{4}$-treated mice and transfected these cells with miR-378a-3p mimic or scrambled-miR (negative control), and then analysed the expression levels of genes related to HSC activation at 24 and $48 \mathrm{~h}$ after transfection. As expected, Gli3 at both the mRNA and protein levels was significantly downregulated in miR-378a-3p mimic-transfected cells, as analysed by qRT-PCR and western blotting (unpaired two-sample Student's $t$-test, $P<0.05$ ) (Fig. $4 \mathrm{a}-\mathrm{c}$ ). Although miR-378a-3p was shown to be unbound to the gli2 mRNA (Fig. 3c), the miR-378a-3p mimic also reduced the expression of Gli2 at $24 \mathrm{~h}$, implying that miR-378a-3p was indirectly regulating Gli2 expression through the interaction between Gli3 and Gli2 (Fig. $4 \mathrm{a}-\mathrm{c}$ ). In addition, in primary aHSCs transfected with a miR-378a-3p mimic, expression of the profibrotic genes encoding for Vimentin, $\alpha$-SMA, Col1 $\alpha 1$ and Mmp-9 decreased, whereas expression of glial fibrillary acidic protein $(g f a p)$, an inactivation marker of HSCs ${ }^{16,24}$, increased (Fig. 4d). In LX2 cells transfected with miR-378a-3p mimic, similar results were observed; the level of Gli3 and Gli2 was reduced and the expression of profibrotic markers, vimentin, plasminogen activator inhibitor-I (pai-I) and connective tissue growth factor (ctgf) was also downregulated ${ }^{25}$, but $g f a p$ was upregulated (Supplementary Fig. 7). Therefore, these results demonstrate that miR-378a-3p suppresses the activation of HSCs by directly targeting gli3.

Smo regulates expression of miR-378. As miR-378a-3p targets Gli3 and the Glis are activated by stimulated Hh receptors, it is possible that Smo influences expression of miR-378a-3p. To investigate this possibility, we treated fully activated LX2 cells with GDC-0449, an Smo antagonist, for 12, 24 and $48 \mathrm{~h}$. As expected, expression of the $\mathrm{Hh}$ receptors, $p t c$ and $s m o$, and the $\mathrm{Hh}$ target genes, gli1, gli2 and gli3, decreased in LX2 cells treated with GDC-0449. The fibrotic markers, tgf- $\beta, \alpha$-sma and coll $\alpha 1$, were also reduced in GDC-0449-treated LX2 cells (Fig. 5a). Interestingly, inhibition of Smo induced the upregulation of pri-miR$378 \mathrm{a}$ and miR-378a-3p at 12 and $24 \mathrm{~h}$ post GDC-0449 treatment (Fig. 5b,c). As this inhibitory effect of GDC-0449 was alleviated, the level of smo gradually increased. In parallel with Smo elevation, the expression of pri-miR-378a and miR-378a-3p in 
a

$$
\begin{aligned}
& \text { miR-378 } \\
& \text { Mouse Gli2 3' UTR } \\
& \text { Human GLI2 3' UTR } \\
& 1,527: 5 \\
& \text { 3' GGAAGACUGAGGU } \\
& \text { ||| |||। } \\
& \text { UCAGGUCA } 5^{\prime} \\
& \text { | | | | | | | }
\end{aligned}
$$

b

Vector constructs
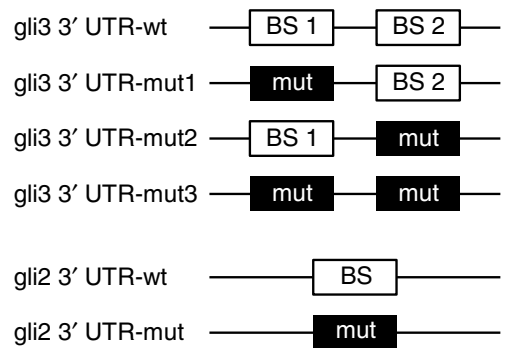

C



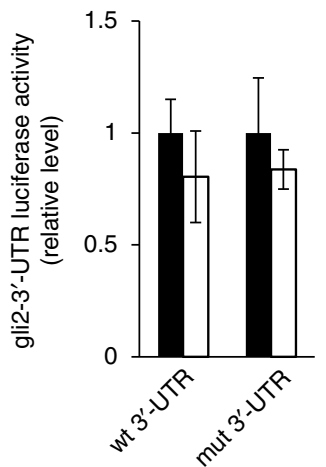

- Scr-miR

口 miR-378a-3p mimic

Figure 3 | MiR-378 binds directly to Gli3. (a) Using an miRNA database (www.miRNA.org), putative binding sites (red font) of miR-378a-3p were predicted in the $3^{\prime}$-UTR of gli2 and gli3 mRNA in mouse and human. The dashed line represents complementary base pairs between miR-378a-3p and gli2 or gli3 mRNA, whereas the grey shading indicates the seed sequence of miR-378a-3p. (b) psiCHECK-2 vectors containing either the wild-type (wt) or mutant (mut) binding site of miR-378a-3p in gli2 and gli3 mRNAs were constructed to conduct luciferase reporter assays. The mutated nucleotides are shown in Supplementary Fig. 6. (c) Dual luciferase reporter assay was performed to verify binding between miR-378a-3p and gli2 or gli3 mRNA. N2a, a mouse neuroblastoma cell line, was co-transfected with a psiCHECK-2 vector containing either the wt or mut target sites plus either the miR-378a-3p mimic or the scrambled (Scr)-miR (control) oligonucleotide. Results of relative luciferase activity are shown as mean \pm s.e.m. obtained from triplicate experiments (unpaired two-sample Student's $t$-test, ${ }^{\star} P<0.05$, ${ }^{\star \star} P<0.005$ versus Scr-miR).

GDC-0449-treated LX2 cells gradually decreased and was similar with it in vehicle-treated LX2 cells. The expressional changes of precursor and mature form of miR-378b and miR-378d were subtle or rare in Smo inhibitor-treated cells, indicating that Smo poorly influenced their expression. As these miRNA are located on different chromosomes, they seem to show the different response to Smo inhibitor.

This elevated expression of pri-miR-378a by Smo suppression suggested that Smo might influence the level of pri-miR-378a transcription. Hence, using a prediction system, the TRANSFAC programme, we examined whether Smo might bind the gene of $m i R-378 a$. The data indicated that an upstream sequence of $m i R-378 a$, from nucleotides -820 to around -811 , contained a putative binding site for NF- $\mathrm{BB}$ (p65), rather than Smo, at the $5^{\prime}$-region (Fig. 6a). Luciferase reporter assay was conducted to assess the functional binding of p65 in this region of the miR$378 a$ gene. We constructed a pGL3 vector with $(+\mathrm{p} 65 \mathrm{BS})$ or without $(\Delta \mathrm{p} 65 \mathrm{BS})$ a p65-binding site and co-transfected HepG2 cells with this vector and either a CDH-p65 vector or a CDH-GFP (control) vector. Luciferase activity was lower in + p65BStransfected HepG2 cells with CDH-p65 than with CDH-GFP vector. However, there was no significant difference in luciferase activity between $\Delta \mathrm{p} 65 \mathrm{BS} / \mathrm{CDH}-\mathrm{GFP}$-transfected and $\Delta \mathrm{p} 65 \mathrm{BS} /$ CDH-p65-transfected HepG2 cells. Thus, the results of the luciferase reporter assay confirmed that p65 directly binds to the $m i R-378 a$ gene (Fig. 6a).

To investigate whether activation of NF- $\kappa \mathrm{B}$ signalling influences expression of miR-378a-3p, we treated cells with either an
NF- $\kappa \mathrm{B}$ signalling activator or repressor. Treatment with tumour necrosis factor $\alpha$ (TNF- $\alpha$ ), an activator of NF- $\kappa B$ signalling, induced upregulation of $p 65$ but downregulation of pri-miR-378a and miR-378a-3p in HepG2 cells (Fig. 6b). In TNF- $\alpha$-treated LX2 cells, expression of miR-378a-3p was not significantly changed (data now shown). As miR-378a-3p is greatly downregulated in LX2 cells, the inhibitory effect of p65 in miR-378a-3p expression seems to be poor. However, the expression level of pri-miR-378a and miR-378a-3p was higher in both LX2 and HepG2 cells treated with the NF- $\kappa$ B inhibitor, Bay 11-7085, than in vehicletreated cells (Fig. 6c). When miRNA expression is simultaneously induced, the level of pri-miRNAs reaches its peak earlier than mature miRNAs do ${ }^{26}$. Similar to these findings, pri-miR-378a in $\mathrm{NF}-\kappa \mathrm{B}$ inhibitor-treated LX2 or HepG2 cells was more upregulated compared with miR-378a-3p at $24 \mathrm{~h}$. These data suggest that p65 functions as a repressor of transcriptional regulation of miR-378a-3p.

To examine how Smo is associated with p65 signalling in the transcriptional regulation of miR-378a-3p, we assessed whether p65 is activated by Smo. The level of p65 protein declined in LX2 cells at 24 and $48 \mathrm{~h}$ post GDC-0449 treatment (Fig. $6 \mathrm{~d}, \mathrm{e})$. TNF- $\alpha$ treatment increased the level of the nuclear fraction of p65 in LX2 cells, whereas TNF- $\alpha$ added to the Smo inhibitor-treated LX2 cells led to a reduction in p65 expression (Fig. 6f,g). The level of the cytosolic p65 was slightly reduced in Smo inhibitor and TNF$\alpha$-treated LX2 cells, indicating that the amount of p65 protein was generally regulated in these cells. The similar results were also observed in HepG2 cells (Fig. 6f,g). Therefore, these results 

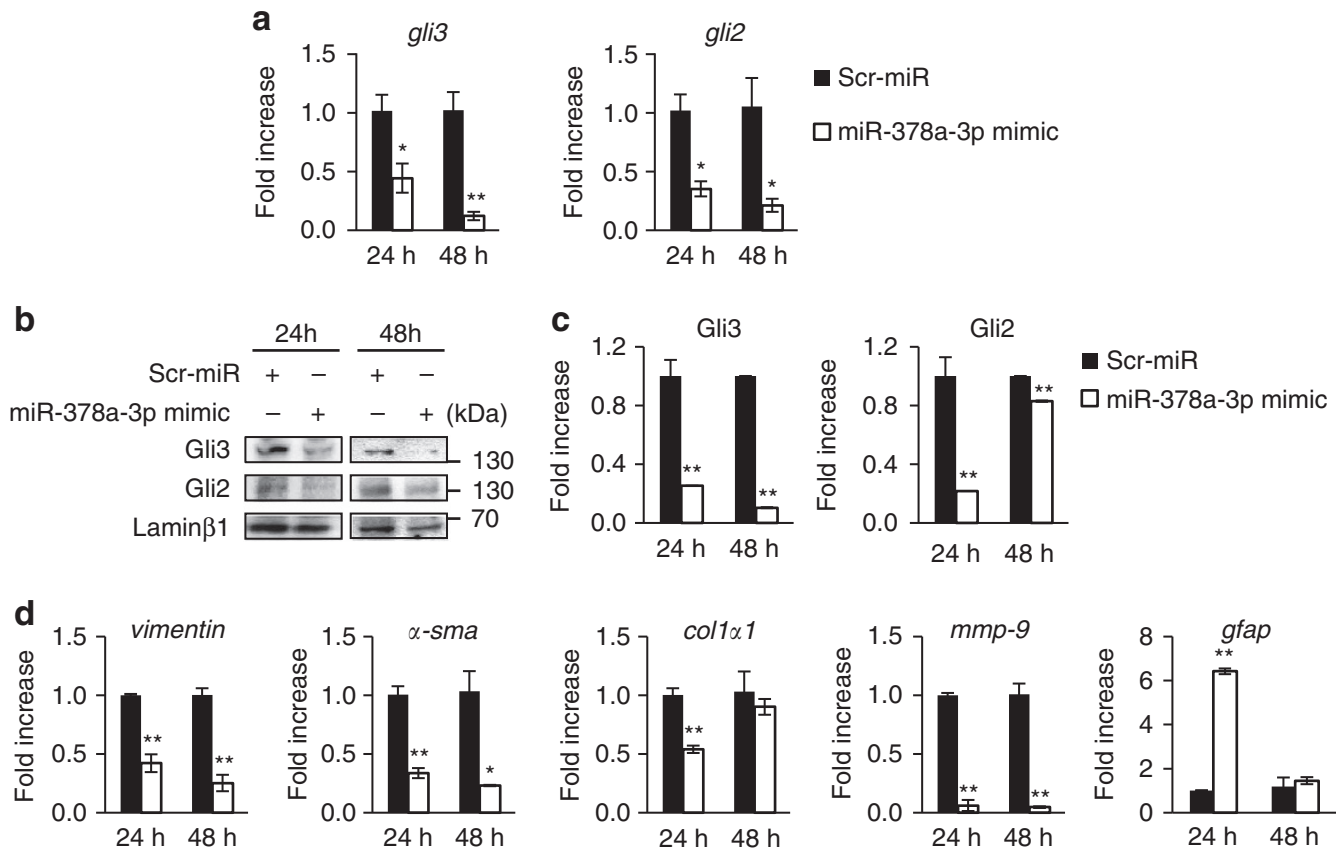

Figure 4 | MiR-378a-3p induces inactivation of primary HSCs by reducing Gli3 and Gli2 expression. (a) Primary aHSCs were transfected with either an miR-378a-3p mimic (white bar) or scrambled ( $\mathrm{Scr}$ )-miR (control) (black bar) oligonucleotide for 24 and $48 \mathrm{~h}$, and expression of gli3 and gli2 was assessed by qRT-PCR. (b) Western blot analysis and (c) cumulative densitometric analyses for nuclear Gli3 (145 kDa) and Gli2 (133 kDa), with Lamin $\beta 1$ (68 kDa) as an internal control for nuclear fraction. Data shown represent one of three experiments with similar results. (d) qRT-PCR analysis for genes related to activation of HSC, including vimentin, $\alpha$-sma, col1 $\alpha 1$ and mmp9, and the inactivation marker of HSC, gfap, in primary HSCs transfected with miR-378a-3p mimic (white bar) or scrambled ( $\mathrm{Scr}$ )-miR (control) (black bar) oligonucleotide for 24 and $48 \mathrm{~h}$. All results of relative expression values are shown as mean \pm s.e.m. obtained from triplicate experiments (unpaired two-sample Student's $t$-test, ${ }^{\star} P<0.05$ and ${ }^{\star \star} P<0.005$ versus Scr-miR).

indicate that Smo promotes nuclear localization of p65, which then binds to the $m i R-378 a$ gene and inhibits transcription of $m i R-378 a$ to pri-miR-378a, suggesting that reduction of miR$378 \mathrm{a}-3 \mathrm{p}$ expression by Smo-activated p65 contributes to increased expression of Gli3 in aHSCs. In previous research, we showed that $\mathrm{Hh}$ signalling was associated with radiation-induced hepatic fibrosis, which was attenuated in GDC-0449-treated liver with irradiation ${ }^{27}$. Hence, we checked the expression of miR-378a-3p in the liver of Smo-suppressed mice with radiation. Expression of $p 65$ decreased in the radiation-treated liver with Smo inhibition, but its expression increased in the radiationtreated liver with fibrosis. Both of pri-miR-378a and miR-378a-3p were downregulated in radiation-treated liver, whereas they were upregulated in irradiated liver treated with Smo inhibitor. Compared with hepatic level of gli3 in irradiated mice, livers of irradiated mice treated with Smo inhibitor contained lower level of gli3 (Supplementary Fig. 8). These results supported the regulatory effect of Smo in the expression of miR-378a-3p through p65 activation.

NP-encapsulated miR-378a-3p reduces hepatic damage. To deliver the miR-378a-3p into experimental animal model, LTU2a NPs encapsulated with miR-378a-3p mimic (NP/M) or scrambled miRNAs (NP/NC) as a negative control were prepared. Transmission electron microscopy of NPs shows relatively spherical morphology and their average sizes are 340 and $516 \mathrm{~nm}$ for NP/M and NP/NC, respectively (Supplementary Fig. 9a,b). Zeta potential analysis showed that the overall charge of them was -9.3 and $-3.9 \mathrm{mV}$ at peak for $\mathrm{NP} / \mathrm{M}$ and $\mathrm{NP} / \mathrm{NC}$, respectively (Supplementary Fig. 9c). In vitro release kinetics show burst release of miRNA for the first 2 days for both formulations
(Supplementary Fig. 9d). Afterwards, the NP/M formulation shows a biphasic release profile, whereas the NP/NC does a controlled release phase. The release of miRNAs reached steady state within 5 weeks. To investigate the uptake of the NPs, LX2 cells were cultured in medium and exposed NPs encapsulated with fluorescein isothiocyanate (FITC)-conjugated BSA for overnight. The co-localization of the NPs within the cell by fluorescence and confocal microscopy (Supplementary Fig. 10) demonstrated successful uptake.

To investigate the function of miR-378a-3p on liver fibrosis in vivo, we first examined the effect of NPs in the healthy livers. Mice were given once i.p. injection of $6 \mathrm{nmol}$ per mice of NP/NC $(n=4)$ or PBS vehicle $(n=4)$ for 3 weeks and then killed. In the examination of liver of mice with or without NPs/NC, liver morphology and expression of genes, $m i R-378 a-3 p$, gli3 and coll $\alpha 1$, were similar between two groups (Supplementary Fig. 11a,b). The expression of $\alpha$-SMA was hardly detected, but the expression of GFAP was greater in both vehicle and NP/NC groups compared with the $\mathrm{CCl}_{4}$-treated group (Supplementary Fig. 11c,d). Based on this data, livers of mice with NPs/NC were used for control group in further analysis. NPs/M or NPs/NC were injected via i.p. route of administration into $\mathrm{CCl}_{4}$ or corn-oil-treated mice. Mice were killed at 1, 2 and 3 weeks after NPs treatment ( $n=4$ per group per week) (Supplementary Fig. 12a). H\&E staining showed the severe hepatic injuries in $\mathrm{CCl}_{4}$-treated mice with $\left(\mathrm{CCl}_{4}+\mathrm{NPs} / \mathrm{NC}\right.$ group $)$ or without $\mathrm{NPs} / \mathrm{NC}\left(\mathrm{CCl}_{4}\right.$ group$)$ at 3 weeks post NP injection. Interestingly, those abnormal morphological changes were remarkably ameliorated in the $\mathrm{CCl}_{4}+\mathrm{NPs} / \mathrm{M}$ group, compared with the $\mathrm{CCl}_{4}$ and $\mathrm{CCl}_{4}+\mathrm{NPs} / \mathrm{NC}$ groups (Supplementary Fig. 12b). The ratio of $\mathrm{LW} / \mathrm{BW}$ of the $\mathrm{CCl}_{4}$ and the $\mathrm{CCl}_{4}+\mathrm{NPs} / \mathrm{NC}$ group increased at 3 weeks, compared with control and the $\mathrm{CCl}_{4}+\mathrm{NPs} / \mathrm{M}$ group 
a
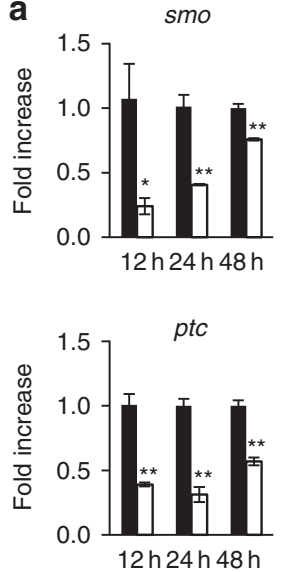

b

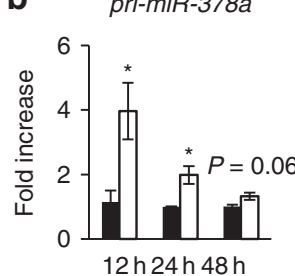

C

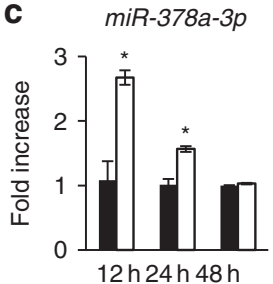

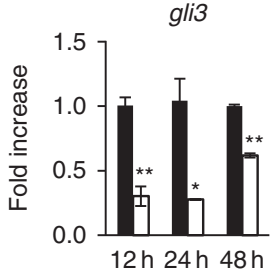

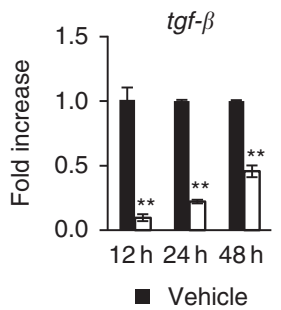

- Vehicle

pri-miR-378b

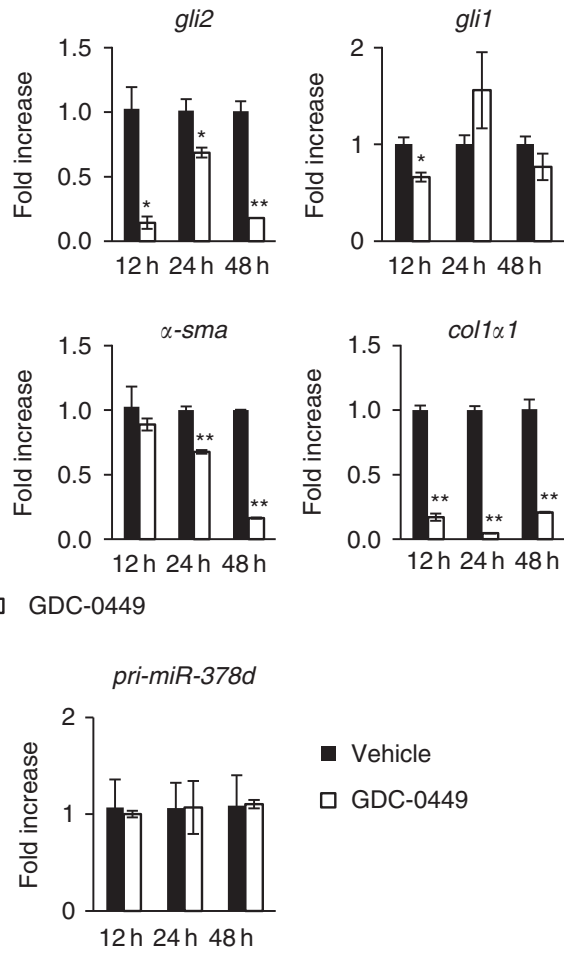

- Vehicle

GDC-0449

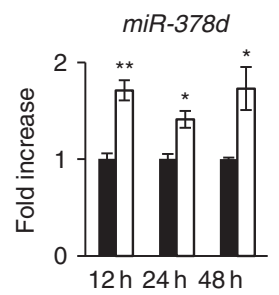

- Vehicle

口 GDC-0449

Figure 5 | Smo influences expression of miR-378. (a) qRT-PCR of the expression of Hh signals, including smo, gli2, gli3, gli1 and ptc, and profibrotic genes, including tgf- $\beta, \alpha$-sma and col1 $\alpha$, in LX2 cells treated with (white bar) or without (black bar) $1 \mu$ M of GDC-0449, a Smoothened (Smo) antagonist, for 12, 24 and $48 \mathrm{~h}$. (b) qRT-PCR analysis of miR-378a precursor (pri-miR-378a), miR-378b precursor (pri-miR-378b) and miR-378d precursor (pri-miR-378d) in LX2 cells treated with (white bar) or without (black bar) GDC-0449 for 12, 24 and $48 \mathrm{~h}$. (c) qRT-PCR analysis of mature family members, including miR-378a3p, miR-378b and miR-378d, in LX2 cells treated with (white bar) or without (black bar) GDC-0449 for 12, 24 and 48 h. All results of relative expression values are shown as mean \pm s.e.m. obtained from triplicate experiments (unpaired two-sample Student's $t$-test, ${ }^{\star} P<0.05$ and ${ }^{\star \star} P<0.005$ versus vehicle).

$\left(\mathrm{CCl}_{4}: 1.31 \pm 0.028, \mathrm{CCl}_{4}+\mathrm{NPs} / \mathrm{NC}: 1.31 \pm 0.019, \mathrm{CCl}_{4}+\mathrm{NPs} / \mathrm{M}:\right.$ $1.17 \pm 0.044$ versus CON, Kruskal-Wallis test and unpaired twosample Student's $t$-test, ${ }^{\star} P<0.05$ and ${ }^{* *} P<0.005$ ) (Supplementary Fig. 12c). In addition, $\mathrm{CCl}_{4}$-treated mice with or without NPs/NC had elevated serum alanine transaminase (ALT) and aspartate transaminase (AST), whereas $\mathrm{CCl}_{4}$-treated mice with NPs/M had alleviated ALT and AST at 3 weeks (ALT: $\mathrm{CCl}_{4}: 2.28 \pm 0.255$, $\mathrm{CCl}_{4}+\mathrm{NPs} / \mathrm{NC}: 2.98 \pm 0.449, \mathrm{CCl}_{4}+\mathrm{NPs} / \mathrm{M}: 1.23 \pm 0.176$ versus CON/AST: $\mathrm{CCl}_{4}: 1.45 \pm 0.083, \mathrm{CCl}_{4}+\mathrm{NPs} / \mathrm{NC}: 1.47 \pm 0.118$, $\mathrm{CCl}_{4}+\mathrm{NPs} / \mathrm{M}: 1.04 \pm 0.159$ versus $\mathrm{CON}$, Kruskal-Wallis test and unpaired two-sample Student's $t$-test, ${ }^{\star} P<0.05$ and ${ }^{* *} P<0.005$ ) (Supplementary Fig. 12d). These results suggested that miR-378a-3p mimic ameliorated chronic liver injury induced by $\mathrm{CCl}_{4}$ in mice.

MiR-378a-3p reduces expression of Gli3 and fibrosis in the liver. To assess whether NPs/M were delivered into livers, the level of hepatic miR-378a-3p was examined by qRT-PCR analysis. Compared with $\mathrm{CCl}_{4}$-treated mice with or without $\mathrm{NPs} / \mathrm{NC}$, expression of miR-378a-3p was significantly upregulated in $\mathrm{CCl}_{4}$-treated mice with NPs/M-injection and gradually increased after NPs/M treatment, suggesting that NPs/M migrated into and stably released RNA oligonucleotides in the liver during this period (Kruskal-Wallis test and unpaired two-sample Student's $t$-test, $P<0.05)$ (Fig. 7a). In addition, expression of miR-378a-3p was higher in primary HSCs isolated from livers of mice treated with $\mathrm{CCl}_{4}+\mathrm{NPs} / \mathrm{M}$ than in primary aHSCs (day 7) and almost similar to in primary qHSCs (day 0) (Fig. 7b). These findings indicated that NPs/M was successfully taken by $\mathrm{HSC}$ in $\mathrm{CCl}_{4}$-injured livers. As most upregulation of miR-378a-3p was observed in $\mathrm{CCl}_{4^{-}}$ treated liver at 3 weeks post NPs/M treatment, we assessed the expression of Gli3 and genes related with HSC activation at this time point. The level of Gli3 in both RNA and nuclear-fractionized protein was downregulated in the $\mathrm{CCl}_{4}+\mathrm{NPs} / \mathrm{M}$ group, compared with the $\mathrm{CCl}_{4}$ or the $\mathrm{CCl}_{4}+\mathrm{NPs} / \mathrm{NC}$ group (Fig. $7 \mathrm{c}-\mathrm{e}$ ). RNA expression of $\alpha$-sma and vimentin, coll $\alpha 1$ and timp1 was reduced in livers of the $\mathrm{CCl}_{4}+\mathrm{NPs} / \mathrm{M}$ group (Fig. 8a). In addition, protein level of $\alpha$-SMA was lower but level of GFAP, inactivation marker of HSC, was higher in the $\mathrm{CCl}_{4}+\mathrm{NPs} / \mathrm{M}$ group than in the $\mathrm{CCl}_{4}$ or the $\mathrm{CCl}_{4}+\mathrm{NPs} / \mathrm{NC}$ group (Fig. 8b,c). Furthermore, immunostaining for $\alpha$-SMA confirmed that accumulation of $\alpha$-SMA-positive HSCs was ameliorated remarkably in the liver of NPs/Mtreated mice with $\mathrm{CCl}_{4}$ treatment (Fig. 8d). These results 
a

Consensus p65 BS: GGGRNNYYCC Putative p65 BS: $\underline{\text { CGGAGTTTCC }}$
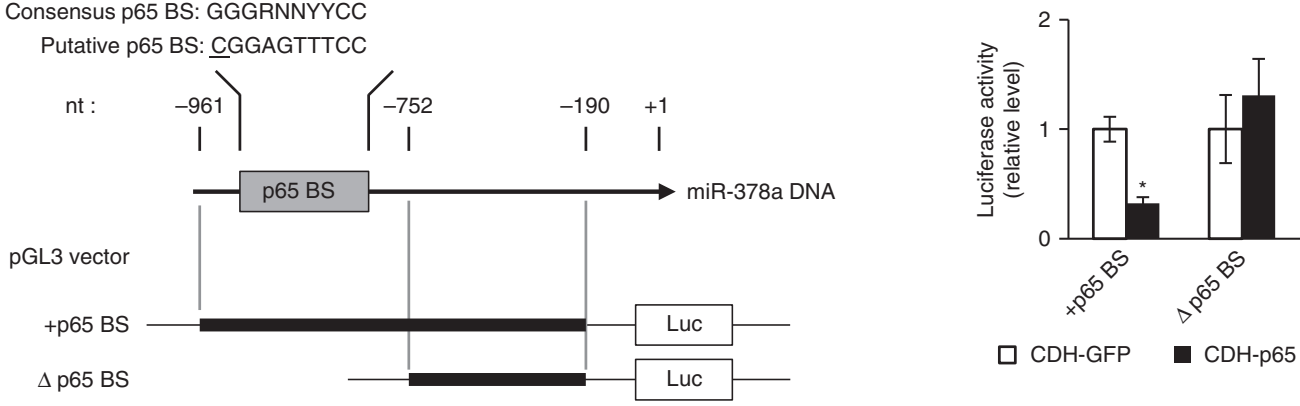

b
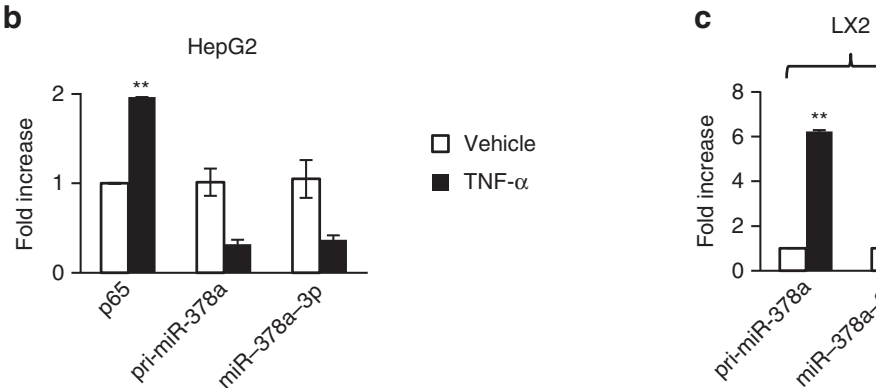

$\square \mathrm{CDH}-\mathrm{GFP}$

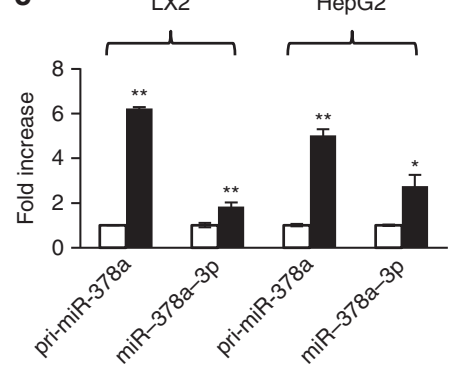

$\square$ Vehicle

Vehicle

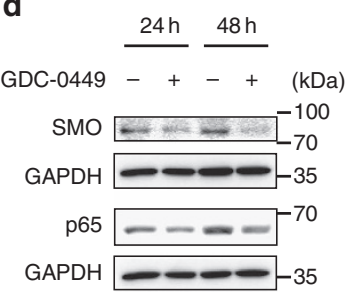



$\mathbf{f}$

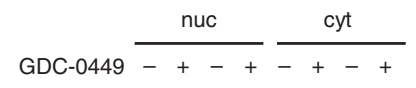

TNF- $\alpha-\ldots++-++$ (kDa)

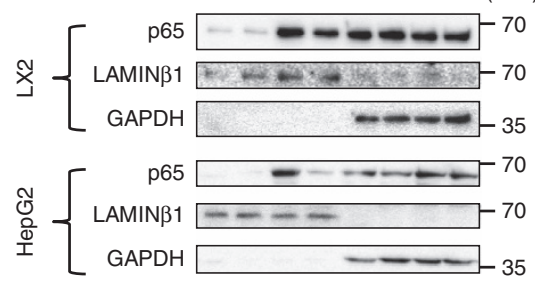

g

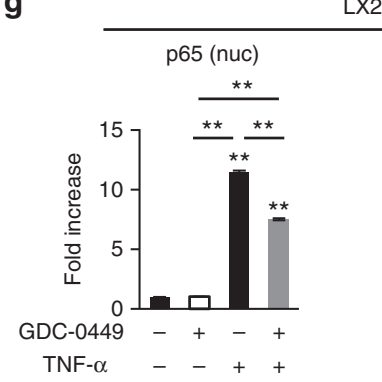

LX2

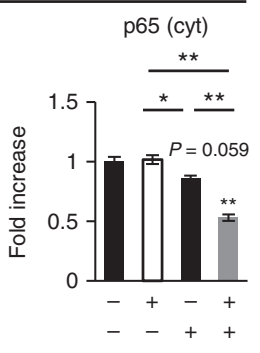

HepG2

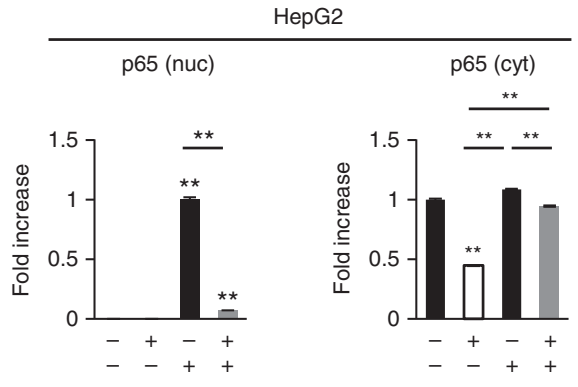

Figure 6 | Expression of miR-378a is regulated by Smo-dependent activation of p65. (a) p65 binding site (p65BS, grey box) on miR-378a DNA (arrow line) and inserted regions (bold line) into pGL3-basic vector are depicted. The consensus and putative binding sequences predicted by TRANSFAC are shown; underlining indicates a mismatched nucleotide (nt). pGL3-basic vectors with ( + p65BS) or without $(\Delta \mathrm{p} 65 \mathrm{BS})$ a p65-binding site on the promoter region of pri-miR-378a were constructed. HepG2 cells were co-transfected with + p65BS and either CDH-p65 (black) or CDH-GFP (control) (white), and a separate group of HepG2 cells were co-transfected with $\triangle \mathrm{p} 65 \mathrm{BS}$ and either $\mathrm{CDH}-\mathrm{p} 65$ (black) or CDH-GFP (white). Results of relative luciferase activity are shown as mean \pm s.e.m. obtained from triplicate experiments (unpaired two-sample Student's $t$-test, ${ }^{\star} P<0.05$ versus CDH-GFP). Luc, luciferase.

(b) Expression level of $p 65$ mRNA, pri-miR-378a and miR-378a-3p in HepG2 treated with $100 \mathrm{ng} \mathrm{ml}^{-1}$ of TNF- $\alpha$ (black) or vehicle (white) was analysed by qRT-PCR. Relative expression is shown as mean \pm s.e.m. obtained from triplicate experiments, compared with HepG2 treated with vehicle (unpaired twosample Student's $t$-test, ${ }^{\star} P<0.05$ and ${ }^{\star \star} P<0.005$ versus vehicle). (c) qRT-PCR analysis for pri-miR-378a and miR-378a-3p in LX2 or HepG2 cells treated with $2 \mu \mathrm{M}$ of Bay 11-7085 (black) or vehicle (white). Results are shown as mean \pm s.e.m. obtained from triplicate experiments (unpaired two-sample Student's $t$-test, ${ }^{\star \star} P<0.005$ versus vehicle). (d) Western blot analysis for SMO (86 kDa), p65 (65 kDa) and GAPDH (36 kDa) in LX2 cells treated with GDC-0449 $(1 \mu \mathrm{M})$ or vehicle for 24 and $48 \mathrm{~h}$. A representative image from triplicate experiments is shown. (e) Cumulative densitometric analyses of SMO and $p 65$ western blotting results are displayed as the mean \pm s.e.m. (unpaired two-sample Student's $t$-test, ${ }^{\star} P<0.05$ and ${ }^{\star \star} P<0.005$ versus vehicle). (f) Western blot assay for p65, Lamin $\beta 1$ (68 kDa) and GAPDH in vehicle- or TNF- and/or GDC-0449-treated LX2 and HepG2 cells. Lamin $\beta 1$ and GAPDH were used as internal controls for the nuclear (nuc) and cytosolic (cyt) fractions, respectively. Data shown represent one of three experiments with similar results. (g) Cumulative densitometric analyses of SMO and p65 western blotting results are displayed as the mean \pm s.e.m. (one-way analysis of variance (ANOVA) with Tukey corrections, ${ }^{\star} P<0.05$ and ${ }^{\star \star} P<0.005$ versus vehicle-treated control). 
a

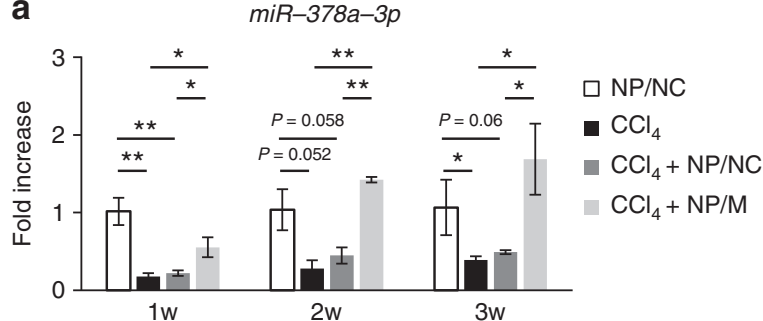

C

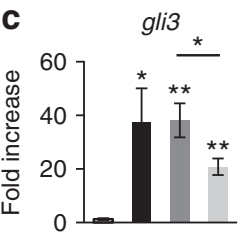

d

d b

$m i R-378 a-3 p$
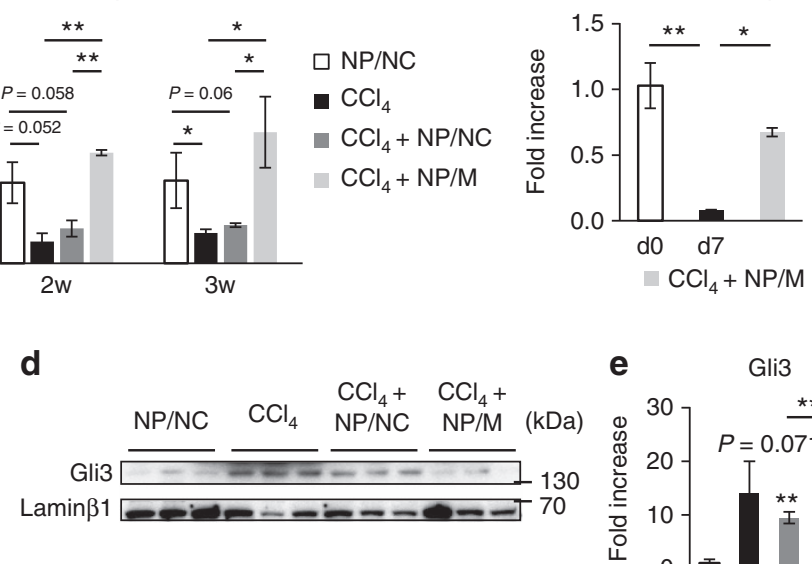

e Gli3

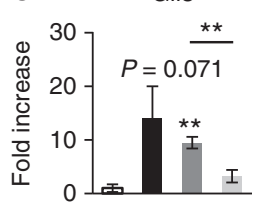

$\square \mathrm{NP} / \mathrm{NC} \square \mathrm{CCl}_{4} \square \mathrm{CCl}_{4}+\mathrm{NP} / \mathrm{NC} \square \mathrm{CCl}_{4}+\mathrm{NP} / \mathrm{M}$

Figure 7 | NPs having miR-378a-3p increase the level of miR-378a-3p but decrease Gli3 expression in CCl $\mathbf{~}_{\mathbf{4}}$-treated mice. (a) qRT-PCR for miR-378a-3p in livers from NP/NC, $\mathrm{CCl}_{4}, \mathrm{CCl}_{4}$-treated with NP/NC $\left(\mathrm{CCl}_{4}+\mathrm{NP} / \mathrm{NC}\right)$ or NP/M $\left(\mathrm{CCl}_{4}+\mathrm{NP} / \mathrm{M}\right)$ mice $(n=4$ per group). Mean \pm s.e.m. results are graphed (Kruskal-Wallis test and unpaired two-sample Student's $t$-test, ${ }^{\star} P<0.05$ and ${ }^{\star \star} P<0.005$ versus NP/NC). (b) qRT-PCR of miR-378a-3p in primary qHSCs isolated from normal mice at quiescent stage (d0; immediately after isolation), primary aHSCs (d7; cultured for 7 days) and primary HSCs isolated from $\mathrm{CCl}_{4}+\mathrm{NP} / \mathrm{M}$ mice at 3 weeks after NPs treatment. Results of relative expression values are shown as mean \pm s.e.m. of triplicate experiments (one-way analysis of variance (ANOVA) with Tukey corrections, ${ }^{\star} P<0.05$ and ${ }^{\star \star} P<0.005$ versus d0). (c) qRT-PCR analysis for gli3 in livers from all mice at 3 weeks after NPs treatment ( $n=4$ per group). Mean \pm s.e.m. results are graphed (Kruskal-Wallis test and unpaired two-sample Student's $t$-test, ${ }^{\star} P<0.05$ and ${ }^{\star \star} P<0.005$ versus NP/NC). (d) Western blot analysis for Gli3 ( $\left.145 \mathrm{kDa}\right)$ and Lamin $\beta 1(68 \mathrm{kDa}$, internal control for nuclear fraction) in livers of three representative mice from each group. Data shown represent one of three experiments with similar results. (e) Cumulative densitometric analyses of Gli3 western blotting results are displayed as the mean \pm s.e.m. (Kruskal-Wallis test and unpaired two-sample Student's $t$-test, ${ }^{\star \star} P<0.05$ ).
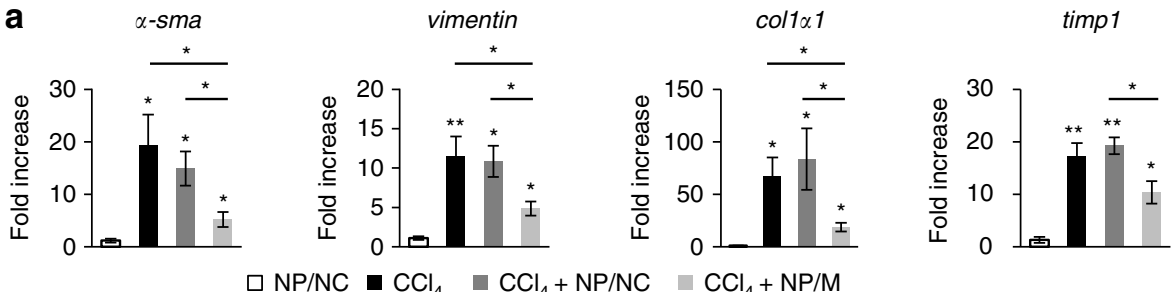

b

$\square \mathrm{NP} / \mathrm{NC} \square \mathrm{CCl}_{4} \quad \square \mathrm{CCl}_{4}+\mathrm{NP} / \mathrm{NC} \square \mathrm{CCl}_{4}+\mathrm{NP} / \mathrm{M}$

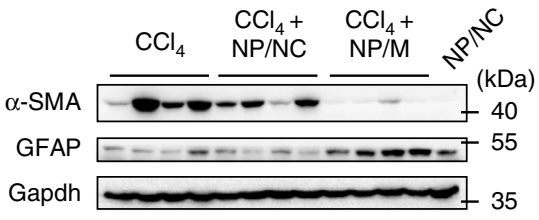

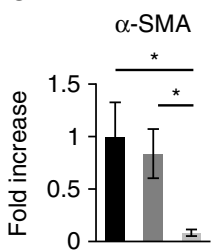

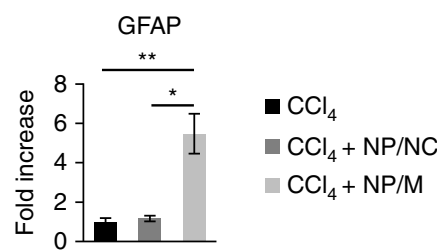

d

NP/NC

$\mathrm{CCl}_{4}$

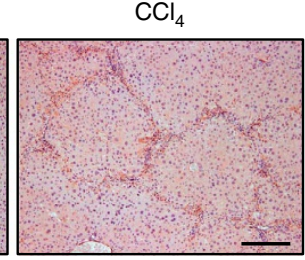

$\mathrm{CCl}_{4}+\mathrm{NP} / \mathrm{NC}$



$\mathrm{CCl}_{4}+\mathrm{NP} / \mathrm{M}$

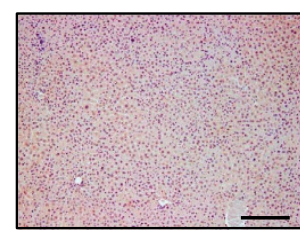

Figure 8 | MiR-378a-3p promotes inactivation of $\mathbf{H S C}$ in $\mathbf{C C l}_{\mathbf{4}}$-treated mice. (a) qRT-PCR analysis for $\alpha$-sma, vimentin, col1 $\alpha 1$ and timp1 in NP/NC, CCl 4 , $\mathrm{CCl}_{4}+\mathrm{NPs} / \mathrm{NC}$ and $\mathrm{CCl}_{4}+\mathrm{NPs} / \mathrm{M}$ group ( $n=4$ per group). All results of relative expression values are shown as mean \pm s.e.m. (Kruskal-Wallis test and unpaired two-sample Student's $t$-test, ${ }^{\star} P<0.05$ and ${ }^{\star \star} P<0.005$ versus NP/NC). (b) Western blot analysis for $\alpha$-SMA (42 kDa), GFAP (50 kDa) and $\mathrm{GAPDH}(36 \mathrm{kDa})$ in livers of representative mice from each group. Data shown represent one of three experiments with similar results. (c) Cumulative densitometric analyses of $\alpha$-SMA and GFAP western blotting results are displayed as the mean \pm s.e.m. (Kruskal-Wallis test and unpaired two-sample Student's $t$-test, ${ }^{\star} P<0.05$ and ${ }^{\star \star} P<0.005$ ). (d) Immunohistochemistry for $\alpha$-SMA in liver sections from representative $N P / N C, C C{ }_{4}, C C l_{4}+N P s / N C$ and $\mathrm{CCl}_{4}+\mathrm{NPs} / \mathrm{M}$ group (scale bar, $100 \mu \mathrm{m}$ ). 
demonstrated that miR-378a-3p delivered by NPs led to downregulation of Gli3, which promoted inactivation of HSCs in the livers of $\mathrm{CCl}_{4}$-treated mice.

Level of miR-378 declines in MCDE-fed mice and human HCC. To assess whether this reduction in levels of the miR-378 family also occurs in other types of liver injury models, we examined the expression of miR-378 family members in livers from mice fed with a methionine/choline-deficient diet supplemented with $0.1 \%$ ethionine (MCDE), which causes the non-alcoholic steatohepatitis (NASH) that accompanies hepatic fibrosis $(n=4$ per group $)^{9}$. Compared with livers of chow-fed mice, livers of MCDE-fed mice contained significantly increased expression of fibrotic markers including vimentin, tgf- $\beta, \alpha$-sma and coll $\alpha 1$ (Fig. 9a). Expression of miR-378a-3p and miR-378d was significantly lower in the MCDE-fed than the normal chow-fed mice at weeks 3 and 4 (unpaired two-sample Student's $t$-test, $\quad P<0.05)$. In addition, miR-378b tended to be downregulated in livers from MCDE-fed mice during liver injury (Fig. 9b).

To investigate the potential role of $\mathrm{miR}-378 \mathrm{a}-3 \mathrm{p}$ in its clinical implications, we analysed the expression of miR-378a-3p and its target, gli3, in non-tumour and tumour regions of liver tissues obtained from 18 patients with hepatocellular carcinoma (HCC) and advanced fibrosis (stage F3 or F4). The level of miR-378a-3p was lower in tumour tissues $(0.82 \pm 0.185)$ than in non-tumour tissues $(2.90 \pm 0.614)$, whereas gli3 mRNA level was higher in tumour tissues $(6.98 \pm 1.991)$ than in non-tumour tissues $(0.76 \pm 0.222)$ (Fig. 9c). As expression of $\mathrm{miR}-378 \mathrm{a}-3 \mathrm{p}$ was decreased in the aHSCs and fibrotic liver tissue containing higher levels of gli3, we performed Spearman's rank correlation analysis to assess the degree of correlation between the levels of miR-378a-3p and gli3 in non-tumour and tumour liver tissue from individual HCC samples. The amount of gli3 mRNA was inversely correlated with the hepatic level of miR-378a-3p in those samples ( $r=-0.434, P=0.008, n=36$ ) (Fig. 9d). These findings support the clinical implications of miR-378a-3p regulating Hh signalling in treating HCC.

\section{Discussion}

MiR-378a is located in the intron of the peroxisome proliferatoractivated receptor- $\gamma$ coactivator $1 \beta$ of human chromosome 5 and mouse chromosome 18. It is co-expressed with its host gene $P G C$ $1 \beta$ and constitutive activation of miR-378a in hepatocytes has been shown to prevent progression to hepatic fibrosis ${ }^{28}$. It was also reported that the miR-378a-3p was abundant in cardiomyocytes, but was downregulated and induced the upregulation of TGF- $\beta 1$ during cardiac fibrosis ${ }^{29}$. In line with the findings of that study, our results demonstrated that expression of miR-378a-3p declined during liver fibrosis. In
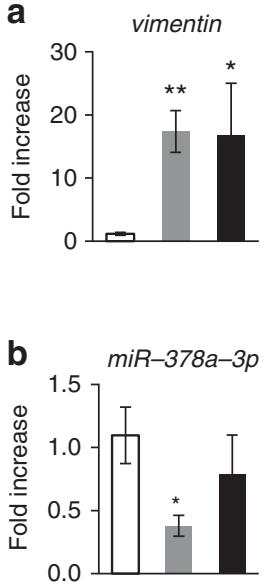

C

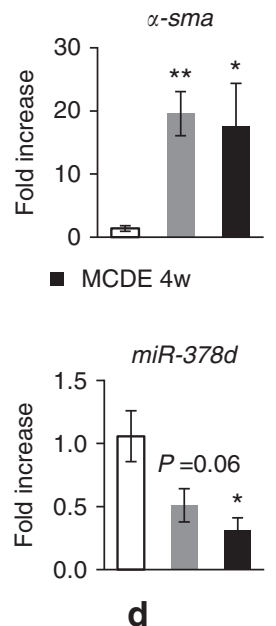

$\square$ CON
MCDE $3 w$
- MCDE $4 w$

d

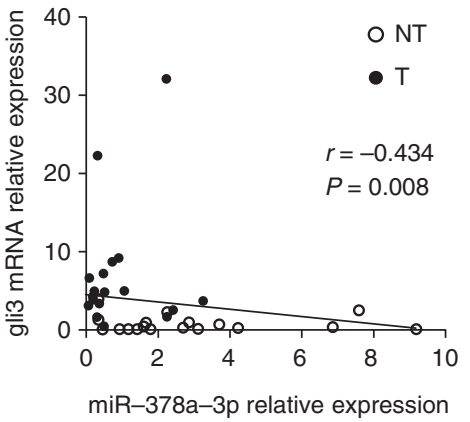

Figure 9 | Expression of miR-378 declines in injured liver of MCDE-fed mice and of patients with HCC. (a) qRT-PCR of pro-fibrotic markers, vimentin, tgf- $\beta, \alpha$-sma and col/ $\alpha 1$, in mice fed with normal chow (CON) or a methionine/choline-deficient diet supplemented with $0.1 \%$ ethionine (MCDE) for 3 and 4 weeks ( $n=4$ per group). Mean \pm s.e.m. results are graphed (unpaired two-sample Student's $t$-test, ${ }^{\star} P<0.05$ and ${ }^{\star \star} P<0.005$ versus CON).

(b) Expression of miR-378 family members, including miR-378a-3p, miR-378b and miR-378d, in MCDE-treated mice was examined ( $n=4$ per group). Mean \pm s.e.m. results are graphed (unpaired two-sample Student's $t$-test, ${ }^{\star} P<0.05$ versus CON). (c) qRT-PCR of miR-378a-3p and gli3 in paired nontumour (NT) and tumour (T) regions of liver tissue from patients with human hepatocellular carcinoma (HCC) ( $n=18$ patients). Individual values represented by the relative expression are shown by dots and their mean \pm s.e.m. results presented for each group (paired two-sample Student's $t$-test, ${ }^{\star} P<0.05$ and ${ }^{\star \star} P<0.005$ versus NT). (d) Spearman's rank correlation between miR-378a-3p expression and gli3 expression in NT and T of HCC (Spearman's rank correlation analysis; $r$, correlation coefficient). 
addition, we found evidence that expression of miR-378a-3p was regulated by Hh/NF- $\kappa \mathrm{B}$ signalling. Supplementary Fig. 13 shows that in qHSCs, the level of miR-378a-3p is high, so that miR378a-3p blocks gli3, contributing to the lower expression of Gli3. When Smo is activated, it promotes the translocation of p65 into the nucleus. This activated p65 binds to the promoter (or control element) of the miR-378a gene, inhibiting its transcription. Reduced miR-378a-3p directly and indirectly increases Gli3 and Gli2, respectively, which accelerate the Hh signalling pathway and the expression of fibrotic genes, eventually promoting liver fibrosis (Supplementary Fig. 13). In addition, the data from the human HCC samples support the correlation between miR-378a$3 \mathrm{p}$ and gli3.

Several factors such as mouse strains, period of $\mathrm{CCl}_{4}$ administration and method of microarray analysis influence the outcomes of microarray analysis ${ }^{30,31}$, although the same chemical, $\mathrm{CCl}_{4}$, is used to induce liver fibrosis. We analysed liver tissues of C57BL/6 mice treated with $\mathrm{CCl}_{4}$ for 10 weeks using SurePrint G3 Mouse miRNA Microarray (Agilent), which covered 1,247 unique mouse miRNA targets using more advanced version (miRBase v19.0). Thus, genome-wide screening of miRNA expression using the more advanced version provided the updated miRNA expression profiles in liver fibrosis and revealed that expression of miR-378 family members including miR-378a-3p, miR-378b and miR-378d were the most significantly reduced among the differentially expressed miRNAs in $\mathrm{CCl}_{4}$-induced liver fibrosis, compared with control. This decreased expression of the miR-378 family was validated in fibrotic livers of mouse models with hepatic injury and also in aHSCs. A previous study also reported that the miR-378 significantly declined in liver tissues of rat with dimethynitrosamine (DMN)-induced hepatic fibrosis ${ }^{32}$.

The miRNA target prediction database (microRNA.org) predicted that the miR-378 family targets human gli3 and gli2 mRNA and mouse gli3 and gli2 mRNA. However, our luciferase reporter assay showed that only miR-378a-3p, and not miR-378b or miR-378d, bound directly to the $3^{\prime}$-UTR region of gli3 mRNA in mouse (Fig. 3 and Supplementary Fig. 4). Mouse miR-378b and miR-378d have a similar sequence and structure to mouse miR-378a-3p, but a different seed sequence. As members of the same miRNA family with different seed sequences bind to different targets ${ }^{33}$, it seems that miR-378b and miR-378d do not bind to the $3^{\prime}$-UTR of gli3 and gli2 mRNA. Although neither miR-378b nor miR-378d binds to gli2 and gli3, expression of both was changed in the injured liver, suggesting that they might have specific roles in the liver. Thus, further study is needed to investigate the functions of these two miRNAs in the liver. In the present study, we focused on the action of miR-378a-3p associated with $\mathrm{Hh}$ signalling.

HSCs are activated during HSC culture $\mathrm{e}^{20,21,34}$. Hh signalling is one of the pathways stimulating the activation and proliferation of HSCs in an autocrine and paracrine manner ${ }^{10-12,16}$. The expression level of Hh signalling is very low in the qHSCs and starts to increase during activation of $\mathrm{HSCs}^{20,35}$. In addition, LSECs produce and respond to $\mathrm{Hh}$ ligands ${ }^{36-38}$, similar to HSCs. However, mature hepatocytes are not Hh-responsive cells, because they do not express Hh target genes such as smo and $\mathrm{Glis}^{35}$. In line with these results, our data showed that members of miR-378 were upregulated in the qHSCs and its' expression in these cells was similar with it in hepatocytes. aHSCs that are positive for Hh signalling showed the decreased expression of these miRNAs. Expression of miR378 was also reduced in Hh-responsive LSECs, compared with both of the qHSCs and the hepatocytes that express lower Hh signalling (Fig. 2). Those findings suggest that the expression level of miR-378 also influences the expression of $\mathrm{Hh}$ signalling in hepatocytes and LSECs, including HSCs, by regulating Gli3 expression.

Our results demonstrate that gli3 is a direct target of miR-378a$3 p$, as shown by bioinformatical analysis and luciferase reporter assay (Fig. 3). Although Gli2, another $\mathrm{Hh}$ target gene, was predicted to be a potential target of miR-378a-3p, no binding interaction between gli2 and miR-378a-3p was observed. However, exogenous expression of miR-378a-3p reduced the expression of Gli3 and Gli2 in LX2 cells, suggesting that downregulation of Gli3 by miR-378a-3p did have an effect on the expression of Gli2, which is in agreement with previous reports that Glis mutually influence each other's expression ${ }^{39,40}$. In addition, gli1 mRNA was downregulated in injured livers compared with normal livers (Supplementary Fig. 5a). In our present study, expression pattern of gli1 mRNA was opposite to the expression of gli2 and gli3 mRNA, which indicated distinct functions of these transcriptional activators (Supplementary Fig. 5a-c). In line with our findings, Pritchett et al. ${ }^{23}$ demonstrated that gli1 was rarely detected in qHSCs and unaltered during activation of HSCs, whereas expression of Gli2 and Gli3 was greatly higher in the aHSCs. In addition, we previously reported a lower expression of gli1 mRNA in livers of rats with fibrosis ${ }^{41}$. In this previous research, we showed that miR-324-5p and miR-326 targeting gli1 mRNA were highly expressed in activated LX2 cells. These results suggest that Gli2 and Gli3 function significantly in liver fibrogenesis.

$\mathrm{NF}-\mathrm{KB}$ signalling regulates expression of genes responsible for immune responses and cell survival ${ }^{42}$. Among the NF- $\mathrm{KBs}$ composed of reticuloendotheliosis (Rel) proteins, including p50, p52, RelA (p65), RelB and c-Rel, which form homodimers or heterodimers, the p50-RelA (also known as p50-p65) heterodimer is the most abundant form of NF- $\mathrm{BB}^{43}$. Classically, $\mathrm{NF}-\kappa \mathrm{B}$ is sequestered in cytoplasm by inhibitors of $\kappa \mathrm{B}$ (IкB), but phosphorylation of I $\mathrm{B}$ by $\mathrm{I} \kappa \mathrm{B}$ kinase allows NF- $\kappa \mathrm{B}$ to translocate into the nucleus and act as a transcription factor ${ }^{42}$ Recent studies have demonstrated that NF- $\mathrm{KB}$ regulates expression of miRNAs ${ }^{44,45}$. Roderburg et al. ${ }^{44}$ reported that NF- $\kappa B-d e p e n d e n t$ downregulation of the miR-29 family promoted the production of ECM proteins in HSCs. In the current study, we found evidence for a new concept that miR$378 \mathrm{a}-3 \mathrm{p}$ and NF- $\mathrm{\kappa B}$ signalling are involved in activation of $\mathrm{Hh}$ signalling during liver fibrosis. Activation of NF- $\kappa B$ by Smo suppressed transcriptional expression of miR-378a-3p and the reduced miR-378a-3p caused an increase in Gli3 and Gli2, which in turn accelerated or promoted the Hh signalling and fibrotic response.

HCC is closely associated with liver fibrosis, as it frequently develops in areas of liver cirrhosis, which is the late stage of liver fibrosis ${ }^{46,47}$. Previous reports have shown that $\mathrm{Hh}$ signalling is strongly expressed in HCC tumour tissue ${ }^{48,49}$. aHSCs, the effector cells of liver fibrosis, promote the development of HCC through their immunomodulatory activities and ECM formation ${ }^{50-52}$. Recent studies report that miR-378a suppresses the growth of hepatitis B virus-related HCC by targeting the insulin-like growth factor 1 receptor $^{53}$. A genetic variant in miR-378a DNA increases its promoter activity so that it acts as a tumour suppressor and leads to decreased susceptibility to HCC in the Chinese population ${ }^{54}$. We investigated expression differences of miR378a-3p and gli3 between tumour and non-tumour liver tissues from patients with HCC and severe fibrosis. We found that expression of miR-378a-3p decreased but expression of gli3 mRNA increased in tumour compared with non-tumour tissues from each HCC sample, demonstrating the correlation of expressional regulation between miR-378a-3p and gli3. In addition, levels of the miR-378 family were also low in injured livers of mice fed for 3 or 4 weeks on an MCDE diet. In the 
MCDE-treated mouse model, the accumulated fatty hepatocytes undergo apoptosis and lead to increased inflammation, proliferation of progenitor cells and activation of HSCs, promoting the progression of liver disease to NASH with fibrosis ${ }^{9}$. Hh signalling is activated and contributes to this fibrogenic response of the liver to injury ${ }^{7,8}$. Hence, it is possible that the miR-378 family is involved in Hh signalling in the MCDE-treated mouse model, indicating that changes in expression of the miR-378 family are relevant to various types of chronic liver diseases. Taken together, our results indicate that miR-378a-3p could be a potential target for diagnosing and treating liver disease with activated Hh signalling.

A few recent studies reported that manipulating the expression of dysregulated miRNAs showed an anti-fibrotic effect in mouse model of $\mathrm{CCl}_{4}$-induced liver fibrosis ${ }^{55,56}$. However, the systemic delivery of RNA-based therapeutics in vivo remains a challenge due to the presence of serum nucleases in blood, the poor uptake by the cells in the targeted tissues and rapid clearance by the renal system $^{68}$. An approach that can minimize these negative effects is nanomedicine. If properly engineered, viral-like features can be mimicked into the design of the NPs such as protection of genetic materials, cellular recognition, assist in the transport of genetic materials across cellular membranes and escape from endosome. In recent times, NPs made from an amino acid-based polymer incorporated with some of these abilities have been shown to effectively transfect tissue in vivo ${ }^{57,58}$. These NPs do not illicit the immune system due to the composition of the polymer and the surface decoration of polyethylene glycol (PEG), which has been shown to increase the circulation time in blood, and also have controlled release capabilities of nucleic acids in the cell's cytoplasm $^{58,59}$. Thus, NPs fabricated with LTU2a, may be an ideal choice for miRNA delivery.

The in vivo results show the potential of NP/M to rescue liver induced with fibrosis even after a single injection. The control release of miR-378a-3p gradually normalizes the ALT and AST levels within 3 weeks after insults with $\mathrm{CCl}_{4}$, even though only $6 \mu \mathrm{g} \mathrm{mg}^{-1}$ of miR-378a-3p were released from the NPs after 1 week. During week 2 and 3, less than half the amount of miR-378a-3p was released but the persistence of release resulted in the gradual rise of the miR-378a-3p levels for each week post NP injection. Thus, the matching of the polymer degradation and release kinetics probably resulted in the normalization of the ALT and AST levels. Furthermore, the recovery of the liver function is observed by the normalization of $\mathrm{Hh}$ signalling, as evidence in the knockdown of Gli3, and matrix production, as shown by decreases in $\alpha$-sma, vimentin, coll $\alpha 1$ and timp1 expressions. These results along with the histology, where the liver injected with NP/M shows similar morphology to the controlled tissues, directly substantiate our hypothesis for the mechanism of liver fibrosis and their potential therapy. Thus, these results merit further development of NPs fabricated with LTU2a as RNA delivery vehicles for nanomedicine.

In conclusion, our study demonstrates that miR-378a-3p suppresses the activation of HSCs by targeting gli3, and that Hh-responsive Smo represses the transcriptional expression of miR-378a-3p through p65, promoting the activation of $\mathrm{Hh}$ and profibrotic genes by increasing the expression of the Hh target genes, Gli2 and Gli3, during liver fibrosis. These findings indicate that miR-378a-3p has great potential to be used as a biomarker of hepatic pathogenesis and a therapeutic agent for treating liver disease.

\section{Methods}

Experimental animal model. Male C57BL/6 mice were purchased from Hyochang (Dae-gu, Korea), housed with 12-h light/dark cycle and allowed free access to normal food and water. To induce liver fibrosis, 7 -week-old mice received
$0.6 \mathrm{ml} \mathrm{kg}^{-1}$ body weight of $\mathrm{CCl}_{4}$ (Sigma-Aldrich, St Louis, MO, USA) dissolved in corn oil by i.p. injection, twice a week for $6(n=5)$ and 10 weeks $(n=6)^{44}$. As a control, same number of mice was injected with equal volume of corn oil. All mice were killed, to obtain serum and liver sample, at $48 \mathrm{~h}$ post the last injection of $\mathrm{CCl}_{4}$ or corn oil. To examine the effect of miR-378 in vivo, 6-week-old mice received $0.4 \mathrm{ml} \mathrm{kg}^{-1}$ body weight of $\mathrm{CCl}_{4}$ (Sigma-Aldrich) dissolved in corn oil by i.p. injection, three times a week for 2 weeks ${ }^{60,61}$. Next, mice were randomly divided into three experimental groups, which were treated with $0.4 \mathrm{ml} \mathrm{kg}^{-1}$ body weight of $\mathrm{CCl}_{4}$, twice a weeks for 3 weeks in parallel with once i.p. injection of $\mathrm{PBS}\left(\mathrm{CCl}_{4}\right.$ group) or NP/M ( $6 \mathrm{nmol}$ of miR-378a-3p mimic per mouse) or NP/NC ( $n=4$ per group per week). As a control, the same number of mice was injected with equal volume of corn oil and NP/NC. Mice were killed to obtain serum and liver samples at 1, 2 and 3 weeks after injection of NPs or vehicle (Supplementary Fig. 12a). To induce NASH or control chow, 10-week-old mice were fed MCDE for 3 or 4 weeks ( $n=5$ per group). Animal experiments with Hh inhibitor GDC-0449 (Selleck

Chemicals, Houston, TX, USA) were described in the previous research ${ }^{27}$. Briefly, eight mice were treated with dimethylsulfoxide (DMSO; $n=4$ ) or $25 \mathrm{mg} \mathrm{kg}^{-1}$ GDC-0449 $(n=4)$, to assess the effect of GDC-0449 in the healthy liver. Based on the previous study ${ }^{62}$, we determined the dose of GDC-0449. In the radiation model, GDC-0449 $(n=5)$ or DMSO $(n=4)$ was i.p. injected into mice $2 \mathrm{~h}$ before getting radiation. After irradiation, mice were given a daily dose of $25 \mathrm{mg} \mathrm{kg}^{-1}$ GDC-0449 or DMSO for 6 weeks and then killed. All animal care and surgical procedures were approved by the Pusan National University Institutional Animal Care and Use Committee and were carried out according to the provisions of the National Institutes of Health $(\mathrm{NIH})$ guidelines for the Care and Use of Laboratory Animals.

Human liver sample. Human HCC tissues with hepatitis B were provided by National Biobank of Korea (PNUH, Pusan, Korea $(n=18)$ ). Samples were graded for the stage 4 of fibrosis (F4), except one sample (F3). The paired tumour or non-tumour tissues were surgically resected from livers with HCC and frozen at $-70{ }^{\circ} \mathrm{C}$. The employed samples in these studies were 14 from male and 4 from female patients. In addition, 11 and 7 patients were non-alcohol and alcohol users, respectively. All samples derived from the National Biobank of Korea were obtained with informed consent under institutional review board-approved protocols (PNU IRB/2013_44_BR) and were studied in accordance with the NIH guidelines for human subject research.

miRNA microarray. Total RNA including miRNA was isolated with TRIzol Reagent (Ambion, Life Technologies, Carlsbad, CA, USA) from liver tissues treated with $\mathrm{CCl}_{4}$ or corn-oil for 10 weeks, following the manufacturer's instructions. RNA quantity and quality were determined using a nanodrop (Thermo Scientific, Waltham, MA, USA) and Bioanalyzer (Agilent Inc., Santa Clara, CA, USA). Total RNA (100 ng) was labelled with desalted Cy3 using an miRNA Complete Labeling and Hyb Kit (Agilent) and then hybridized to an Agilent miRNA expression microarray in accordance with the manufacturer's instructions. A SurePrint G3 Mouse miRNA Microarray (Release 19.0, $8 \times 60 \mathrm{~K}$; Agilent) platform was used for miRNA profiling and raw data were extracted using Feature Extraction Software (v11.0.1.1; Agilent). For microarray analysis, raw data were first filtered by a flag signal detected in all samples. Filtered raw data were processed using the Limma Bioconductor package (http://www.bioconductor.org/) in the R statistical environment (http://www.r-project.org/). After quantile normalization of data, miRNAs with twofold or greater differential expression were identified, with $P$-values of $<0.05$ being considered statistically significant.

Cloning of vector constructs. Target genes of human and mouse miR-378 were predicted by bioinformatic analysis using the online database http://www.microrna.org/. Genomic DNA (gDNA) was isolated from normal mouse liver and its concentration and purity examined using Nanodrop (Thermo Scientific). The $3^{\prime}$-UTR of mouse gli3 and gli2, containing binding sites for mouse miR-378, were amplified by PCR using mouse gDNA. The PCR product was purified using AccuPrep PCR Purification Kit (Bioneer, Daedeok-gu, Daejeon, Korea), cut by the restriction enzymes Xhol and Not1, and then cloned into the psiCHECK-2 vector (Promega, Madison, WI, USA). The vector constructs with $3^{\prime}$-UTR of gli3 and gli2 were transformed into Escherichia coli and then plasmid DNA was extracted from well-transformed, ampicillin-resistant E. coli, using an AccuPrep Plasmid Mini Extraction Kit (Bioneer). The sequences of the miR-378-binding sites of the $3^{\prime}$-UTR of gli3 and gli2 were confirmed by sequencing analysis (Macrogen, Seoul, Korea). Mutant vectors lacking the miR-378-binding site were produced by site-directed mutagenesis using a QuikChange Site-Directed Mutagenesis Kit (Stratagene, Agilent) in accordance with the manufacturer's instructions. All primer sequences used for vector construction are listed in Table 1.

Putative binding sites of transcription factors were analysed by TRANSFAC database (assembly Grch37/hg19). According to a database of microRNA TSS (miRStart), transcription start site is resided at $-1,069$ nucleotides (nt) on the $m i R-378 a$ gene (chr5:149112388-149112453). Promoter region from -961 to $-190 \mathrm{nt}$ including binding site of vertebrate p65 $(+$ p65BS $)$ were amplified by PCR using human gDNA. In addition, promoter regions from -752 to $-190 \mathrm{nt}$ excluding p65-binding site were amplified by the same system $(+/ \Delta$ p65BS $)$. 
Table 1 | Primer sequences used for cloning.

\section{Gene}

gli2 wt 3'UTR_F

gli2 wt 3'UTR_R

gli3 wt 3'UTR_F

gli3 wt $3^{\prime}$ UTR_R

gli2 mut $3^{\prime}$ UTR_F

gli2 mut 3'UTR_R

gli3 mut 3'UTR-1_F

gli3 mut 3'UTR-1_R

gli3 mut 3'UTR-2_F

gli3 mut 3'UTR-2_R

+ p65 BS_F

+ p65 BS_R

$\Delta$ p65 BS_F
Primer sequences $\left(5^{\prime}-3^{\prime}\right)$

TTTTCTCGAGTCTCTGGCTCTTGTGGTGTG

TTGCGGCCGCCACCAAATTTACTGCCTGGA

TTTTCTCGAGGAGTCAAAAGTGTTCTATCCCAAGA

TTGCGGCCGCTTCCTGGTGACACTGTCTGG

GTTTGGCAATATAAATTTGGGTCGTCCAAATTTGGATTCACCCACAGTC

GACTGTGGGTGAATCCAAATTTGGACGACCCAAATTTATATTGCCAAAC

CACCAATCTGTCCTGATTGACGACTTCAAGAATGATCATTTGG

CCAAATGATCATTCTTGAAGTCGTCAATCAGGACAGATTGGTG

CAGCCTCTGAGTGCTGACTCCTCAACAACAACAACTGGTCC

GGACCAGTTGTTGTTGTTGAGGAGTCAGCACTCAGAGGCTG

TTTGCTAGCCACTTGCTGCCGTACTTTCA

TTTTCTCGAGCTCCCACTCCAGGTACCAAA

TTTGCTAGCGACAGCGTCTTCCTGGTTTC

F, forward; mut, mutant; R, reverse; wt, wild-type.

Primer sequences shown in this table were used for cloning the vector constructs.

Purified PCR products by AccuPrep PCR Purification Kit (Bioneer) were cut by the restriction enzymes Nhe1 and Xhol, and inserted into the pGL3-basic vector (Promega). The vector constructs with promoter region of primary (pri)-miR-378a were transformed into E. coli and then plasmid DNA was extracted from well-transformed and ampicillin-resistant E. coli using an AccuPrep Plasmid Mini Extraction Kit (Bioneer). The inserted regions in these experiments were confirmed by sequencing analysis (Macrogen). All primer sequences used for vector construction are listed in Table 1.

Isolation of primary liver cells. Primary hepatic cells including hepatocytes, HSCs and LSECs were isolated as described previously ${ }^{63-66}$. Briefly, male C57BL/6 mice weighing 26-30 g were anaesthetized with zoletil $50\left(5 \mathrm{mg} \mathrm{kg}^{-1}\right.$ body weight, Virbac SA, France), to immobilize in the recumbent position on a treatment table, and the inferior vena cava was cannulated under aseptic conditions. Livers were perfused in situ with EGTA and collagenase (Roche, Indianapolis, IN, USA), to disperse the cells. Primary hepatocytes were separated from non-parenchymal cells using Percoll density gradient centrifugation. Isolated hepatocytes were cultured in DMEM medium (Gibco, Life Technologies, Carlsbad, CA, USA) containing 10\% fetal bovine serum (FBS, Gibco, Life Technologies) and 1\% penicillin/streptomycin (P/S, Gibco, Life Technologies). Primary HSCs and LSECs were isolated by differential centrifugation on OptiPrep (Sigma-Aldrich) density gradient and located on the upper layer of $11.5 \%$ and $20 \%$ OptiPrep, respectively. The purity of HSCs was $>98 \%$, as established by microscopy examination for lipid droplets and vitamin A autofluorescence. The upper layer of $20 \%$ OptiPrep contains LSEC and Kupffer cell fraction. As LSECs poorly attach culture plastic dish, LSECs are separated from Kupffer cell fraction by selective adherence. Isolated HSCs and LSECs were cultured in RPMI 1640 medium

(Gibco, Life Technologies) containing $10 \% \mathrm{FBS}$ and $1 \% \mathrm{P} / \mathrm{S}$ at $37^{\circ} \mathrm{C}$ in a humidified atmosphere containing $5 \% \mathrm{CO}_{2}$. As determined by trypan blue exclusion, cell viability was $>92 \%$ in all experiments.

Cell experiments. LX2 cell, human HSC line (provided by Dr Jeong, KAIST, KOREA), HepG2 cell, human hepatocyte cell line (provided by Dr Jang, PNU, KOREA) and N2a cell, mouse neuroblastoma cell line (provided by Dr Youn, PNU, KOREA), were cultured in DMEM supplemented with $10 \% \mathrm{FBS}$ and $1 \% \mathrm{P} / \mathrm{S}$ at $37^{\circ} \mathrm{C}$ in a humidified atmosphere containing $5 \% \mathrm{CO}_{2}$. To evaluate the effect of miR-378 on HSC activation, LX2 cells $\left(1.5 \times 10^{5}\right.$ per well $)$ cultured for $24 \mathrm{~h}$ were transfected with $20 \mathrm{nM}$ of miR-378 mimic (AccuTarget human miRNA-378a-3p mimic, Bioneer) or $20 \mathrm{nM}$ of scrambled miRNA (miRNA mimic negative control 1, Bioneer) as a negative control using Lipofectamine RNAi/MAX transfection reagent (Invitrogen, Life Technologies, Carlsbad, CA, USA), according to the manufacturer's instructions. After $6 \mathrm{~h}$, the medium was changed with fresh medium containing $2 \% \mathrm{FBS}$ and $1 \% \mathrm{P} / \mathrm{S}$, and then these transfected cells were incubated at $37^{\circ} \mathrm{C}$ and $5 \% \mathrm{CO}_{2}$ atmosphere for 24 or $48 \mathrm{~h}$. To inhibit Smo, fully activated LX2 cells were treated with Vismodegib (1 $\mu \mathrm{M}$ of GDC-0449; Selleck Chemicals), Smo antagonist or vehicle (DMSO) for 24 and $48 \mathrm{~h}$. To assess the regulation of pri-miR-378a expression by NF- $\mathrm{kB}$, HepG2 cells were treated with TNF- $\alpha\left(100 \mathrm{ng} \mathrm{ml}^{-1}\right)$, activator of NF- $\mathrm{KB}$ signalling or vehicle (PBS), and LX2 and HepG2 cells were treated with Bay 11-7085 $(2 \mu \mathrm{M})$, repressor of NF- $\kappa$ B or vehicle (DMSO) for $24 \mathrm{~h}$. To evaluate the relationship of Smo with NF- $\kappa \mathrm{B}, \mathrm{LX} 2$ and HepG2 cells were treated with GDC-0449 $(1 \mu \mathrm{M})$ for $18 \mathrm{~h}$, followed by addition of TNF- $\alpha\left(100 \mathrm{ng} \mathrm{ml}^{-1}\right)$. These cells were harvested at $6 \mathrm{~h}$ post treatment of TNF- $\alpha$. As determined by trypan blue exclusion, cell viability was $>90 \%$ in all experiments. The data were shown as the mean \pm s.e.m. obtained from three different experiments.
Luciferase reporter assay. One day before transfection, $10^{6} \mathrm{~N} 2 \mathrm{a}$ or HepG2 cells were spread over 24-well culture plates in culture medium without antibiotics. Using Lipofectamine 2000 (Invitrogen), N2a cells were transfected with a mixture of psiCHECK-2 vector construct and either an miR-378a-3p mimic ( $25 \mathrm{nM}$; AccuTarget mouse miRNA-378a-3p mimic; Bioneer) or the same concentration of scrambled miRNA (miRNA mimic negative control 1; Bioneer) as a negative control. HepG2 cells were transfected with a mixture of pGL3-vector construct and CDH-p65 or CDH-GFP (control) using Lipofectamine 2000. CDH-p65 was a CDH-GFP lentiviral vector cloned by $\mathrm{p} 65$ construct $^{67}$. At $24 \mathrm{~h}$ after transfection, cells were harvested and tested with the Dual Luciferase Reporter Assay System (Promega) in accordance with the manufacturer's protocol. All firefly luciferase activity data are normalized to Renilla luciferase activity and presented as the mean \pm s.e.m. of values from at least three repetitive experiments.

RNA analysis. Total RNA was extracted from liver tissues or cells by using TRIzol reagent (Ambion, Life Technologies) or miRNeasy Mini Kit (Qiagen, Valencia, CA, USA) to enrich miRNA quantity. The concentration and purity of RNA were determined using a nanodrop. Template complementary DNA was synthesized from total RNA using the SuperScript First-strand Synthesis System (Invitrogen, Life Technologies) or miScript Reverse Transcriptase Kit (Qiagen) according to the manufacturer's protocols. We performed the real-time qRT-PCR analysis by using Power SYBR Green Master Mix (Applied Biosystem, Life technologies) or miScript SYBR Green PCR Kit (Qiagen) on the manufacturer's specifications (Eppendorf, Mastercycler Real-Time PCR). All reactions were triplicated and data were analysed according to the $\Delta \Delta \mathrm{C}_{\mathrm{t}}$ method. $40 \mathrm{~S}$ ribosomal protein $\mathrm{S} 9 \mathrm{mRNA}$ and $18 \mathrm{~S}$ ribosomal RNA for mRNA and U1A small nuclear RNA (RNU1A) for miRNA were used for normalization of the expression level. In data analysis for the expression of miR-378a-3p and gli3 in human samples, average of expression of miR-378a-3p or gli3 from all paired non-tumour and tumour tissues was calculated. Next, individual value of expression relative to the mean value was presented as scatter plot and mean \pm s.e.m. of each group was graphed. The sequences of all primers used in this study are summarized in Supplementary Tables 1 and 2. All PCR products were directly sequenced for genetic confirmation (Macrogen).

Western blotting. Total protein was extracted from frozen liver tissues stored at $-80^{\circ} \mathrm{C}$ and cultured cells. Samples were homogenized in triton lysis buffer supplemented with protease inhibitors (Roche) and centrifuged at 13,000 r.c.f. for $15 \mathrm{~min}$. The supernatants containing protein extracts were used in subsequent biochemical analysis. Protein concentration was measured by Pierce BCA Protein Assay Kit (Thermo Scientific). Equal amount of total protein lysates were separated by 8 or $10 \%$ SDS-PAGE and then transferred onto a polyvinylidene difluoride membrane (Millipore, Darmstadt, Germany). Primary antibodies used in this study were as follows: rabbit anti-Smo antibody (diluted 1:1,000; Abcam), rabbit antiGli2 antibody (diluted 1:1,000; GenWay Biotech, Inc., San Diego, CA, USA), rabbit anti-Gli3 antibody (diluted 1:1,000; Abcam), rabbit anti-transforming growth factor- $\beta$ antibody (diluted 1:1,000; Cell Signaling Technology, Inc., Danvers, MA, USA), mouse anti- $\alpha$-SMA antibody (diluted 1:1,000; Sigma-Aldrich), rabbit antip65 antibody (diluted 1:1,000; Santa Cruz Biotechnology, Inc., Santa Cruz, CA, USA), rabbit anti-GFAP antibody (diluted 1:1,000; Dako, Carpinteria, CA, USA) and mouse anti-glyceraldehydes 3-phosphate dehydrogenase antibody (diluted 1:1,000; AbD Serotec, Oxford, UK) as an internal control. Horseradish peroxidaseconjugated anti-rabbit or anti-mouse IgG (Amersham ECL, GE Healthcare, Milwaukee, WI, USA) was used as secondary antibody. Protein bands were detected using an EzWestLumi ECL solution (ATTO Corporation, Tokyo, Japan) as per the manufacturer's specifications (ATTO Corporation, Ez-Capture II). 
Densities of protein bands were measured using CS Analyzer software (Version 3.00.1011, ATTO \& Rise Corporation).

To separate nuclear and cytosolic fraction from total protein, harvested cells were resuspended with buffer A (10 mM HEPES, $50 \mathrm{mM} \mathrm{NaCl}, 1 \mathrm{mM}$ dithiothreitol, $0.1 \mathrm{mM}$ EDTA and $0.1 \mathrm{mM}$ phenylmethylsulfonyl fluoride) and incubated on ice for $20 \mathrm{~min}$. After adding $0.1 \%$ of NP-40, lysated cells were incubated for $20 \mathrm{~min}$ additionally. After centrifugation at 5,000 $\mathrm{g}$ for $2 \mathrm{~min}$, the supernatant was collected for cytosolic fraction. Pellet was resuspended with buffer B (20 mM HEPES, $400 \mathrm{mM} \mathrm{NaCl}, 1 \mathrm{mM}$ dithiothreitol, $1 \mathrm{mM}$ EDTA, $1 \mathrm{mM}$ phenylmethylsulfonyl fluoride and $1 \mathrm{mM} \mathrm{EGTA}$ ) and incubated on ice for $30 \mathrm{~min}$. After centrifugation at 13,000 r.p.m. for $15 \mathrm{~min}$, the supernatant was saved for nuclear fraction. Rabbit-anti-Lamin $\beta 1$ antibody (diluted 1:1,000; Abcam) was used as a marker of nuclear protein and internal control.

Liver histology and immunohistochemical stain. To examine hepatic morphology and assess liver fibrosis, H\&E staining and Sirius red staining were performed, respectively. Liver specimens were fixed in $10 \%$ neutral buffered formalin (Sigma), embedded in paraffin and cut into $4 \mu \mathrm{m}$ sections. Next, the specimens were deparaffinized, hydrated and stained by standard methods.

For immunohistochemistry, liver sections were deparaffinized, hydrated and incubated in 3\% hydrogen peroxide, to block endogenous peroxidase. Antigen retrieval was performed by heating in $10 \mathrm{mM}$ sodium citrate buffer $(\mathrm{pH} 6.0)$ for 10 min using microwave. Specimens were blocked in Protein Block solution (Dako) for $30 \mathrm{~min}$ at room temperature (RT) followed by incubation with primary antibody at $4{ }^{\circ} \mathrm{C}$ overnight. Other sections were also incubated at $4{ }^{\circ} \mathrm{C}$ overnight in non-immune sera. Rabbit $\alpha$-SMA antibody (diluted 1:500; Abcam) was used as a primary antibody and diluted in Protein Diluent (Dako). Polymer-horseradish peroxidase anti-rabbit (Dako) was used as secondary antibody and 3,3' diaminobenzidine as brown colour was used to visualize the protein.

Hydroxyproline assay. Hydroxyproline content of the livers was calculated by the method previously described ${ }^{9,41}$. Briefly, $50 \mathrm{mg}$ of freeze-dried liver tissue was hydrolysed in $6 \mathrm{~N} \mathrm{HCL}$ at $110^{\circ} \mathrm{C}$ for $16 \mathrm{~h}$. The hydrolysate was evaporated under vacuum and the sediment was re-dissolved in $1 \mathrm{ml}$ distilled water. Samples were filtered using $0.22 \mu \mathrm{m}$ filter centrifuge tube at 14,000 r.p.m. for $5 \mathrm{~min}$. Lysates were then incubated with $0.5 \mathrm{ml}$ of chloramines-T solution containing $1.41 \mathrm{~g}$ of chloramine-T dissolved in $80 \mathrm{ml}$ of acetate-citrate buffer and $20 \mathrm{ml}$ of $50 \%$ isopropanol, at RT. After $20 \mathrm{~min}, 0.5 \mathrm{ml}$ of Ehrlich's solution, containing $7.5 \mathrm{~g}$ of dimethylaminobenzaldehyde dissolved in $13 \mathrm{ml}$ of $60 \%$ perchloric acid and $30 \mathrm{ml}$ of isopropanol, was added to the mixture, which was incubated at $65^{\circ} \mathrm{C}$ for $15 \mathrm{~min}$. After cooling to the RT, the absorbance was read at $561 \mathrm{~nm}$. Amount of hydroxyproline in each sample was determined using the regression curve from the hydroxyproline prepared with high purity hydroxyproline (Sigma-Aldrich) and divided by the amount of liver weight contained in the initial sample $(50 \mathrm{mg})$ to get the hydroxyproline contents ( $\mu \mathrm{g}$ hydroxyproline per $\mathrm{mg}$ liver). Data were expressed as fold changes by comparing with hydroxyproline content of the control group.

Measurement of ALT and AST. The levels of serum ALT (GPT, glutamatepyruvate transaminase) and AST (GOT, glutamate-oxaloacetate transaminase) were measured using GOT - GPT measuring reagents (Asan Pharmaceutical, Seoul, Korea) according to the manufacturer's instructions.

Polymer synthesis. A 'pseudo' poly(amino acid)-based polyurethane has been synthesized according to previous established protocol ${ }^{58,68}$. The synthesis of the LTU2a was completed first by the formation of hexyl ester of L-tyrosine. Next, desaminotyrosyl-tyrosine-hexyl ester was synthesized by coupling hexyl ester of L-tyrosine to desamino tyrosine (Sigma-Aldrich) through a carbodiimide reaction. Finally, a pre-polymer of PEG (molecular weight 2,000 Da, Sigma) and hexamethylene diisocynate (Sigma-Aldrich) was formed using stannous octoate (Sigma-Aldrich). The resulting polymer was precipitated in a chilled sodium chloride solution $\left(12 \mathrm{gl}^{-1}\right)$. The product was vacuum dried at $40^{\circ} \mathrm{C}$ for 3 days before characterization. The LTU2a synthesis yielded a polymer with an average molecular weight of $36,270 \mathrm{Da}$ and polydispersity index of 1.6 . Once the product has been verified with nuclear magnetic resonance (NMR) and gel permeation chromatography (GPC), LTU2a was used to make NPs.

NP formulation. NPs were formulated by blending LTU2a (197 $\mathrm{mg}$ dissolved in $9 \mathrm{ml}$ of chloroform), copolymer of PEG-PLA ( $2 \mathrm{mg}$ dissolved in $1 \mathrm{ml}$ of chloroform) and linear polyethylenimine (LPEI) complexed with miRNA (100 nmol of miR-378a-3p or $100 \mathrm{nmol}$ of scrambled miRNA at mass ratios of 5:1) or $2 \mathrm{mg}$ of FITC-BSA (Sigma-Aldrich) dissolved in $1 \mathrm{ml}$ of diethylpyrocarbonate (DEPC)treated water using a water-in-oil-in-water emulsion and solvent evaporation ${ }^{57,58}$. The initial emulsion was formed with a high-speed mixer at 2,000 r.p.m. in a round-bottom glass flask for $3 \mathrm{~min}$. Afterwards, the speed was lowered to 1,600 r.p.m., $5 \%$ polyvinyl alcohol (PVA) (100 ml, Sigma-Aldrich) was added and mixed for an additional minute. The organic solvent was allowed to evaporate overnight. The NPs were washed by centrifugation and lyophilized for 3 days.
NP characterization. Five milligrams of either NP/M or NP/NC was suspended in $5 \mathrm{ml}$ of distilled and deionized water. The $10 \mu \mathrm{l}$ of NP solution was pipetted onto a copper grid. After water evaporation, transmission electron microscopy (JEOL 2010) images were obtained. Dynamic light scattering (DLS) analysis (Nano ZS, Malvern) and zeta potential (Nano ZS, Malvern) were obtained using $1 \mathrm{ml}$ of the NP solution.

miRNA release studies. Release studies of miRNA were performed according to published methods ${ }^{57,59}$. Five milligrams of NP/M or NP/NC was incubated with $1 \mathrm{ml}$ of DEPC-treated water in a shaker bath set at $37^{\circ} \mathrm{C}$. The samples were centrifuged, the supernatant was collected and the NPs were resuspended at days $1,2,4,7,14,21,28,35$ and 42 . The amount of release for miR-378a-3p and scrambled miRNA was determined with Quant-iT PicoGreen assay (Thermo Scientific). Known standards of miRNA-LPEI complexes were used to calculate the release profile of LTU2a-NPs ${ }^{57,58}$.

Uptake studies. LX2 cells were seeded onto German coverslips at $1.5 \times 10^{4}$ cells per $\mathrm{cm}^{2}$ and cultured as previously described. Next day, NPs encapsulated with FITC-BSA were suspended in cell culture medium at $0.1 \mathrm{mg} \mathrm{ml}^{-1}$ and exposed to cells for $\sim 24 \mathrm{~h}$. Next, the cells were fixed with $0.1 \%$ formaldehyde solution in PBS. As an optional procedure, the fixed cells were stained rhodamine phalloidin (Thermo Scientific). The coverslips were mounted with an anti-fade reagent (VectorShield) and imaged with fluorescence (Nikon) or confocal microscopy (Zeiss).

Statistical analysis. Results are expressed as the mean \pm s.e.m. Statistical significances between control and treated groups or subgroups were analysed by the unpaired two-sample Student's $t$-test or one-way analysis of variance followed by a post-hoc Tukey's test. Data for in vivo effect of miR-378 in the liver were first analysed with the non-parametric Kruskal-Wallis test and then the differences between subgroups were further analysed by the two-sample Student's $t$-test. Differences were considered as significant when $P$-values are $<0.05$. Results from HCC patients were expressed as individual values of relative gene expression and the mean \pm s.e.m., to show distributions of most variables in patients. Difference between non-tumour and tumour group was determined using the paired two-sample Student's $t$-test and considered as significant when $P$-values are $<0.05$. The degree of correlation between the expression levels of miR-378a-3p and gli3 in liver tissues of HCC and between the level of miR-378a-3p and hydroxyproline content or Gli3 in liver tissues of $\mathrm{CCl}_{4}$-treated mice were analysed by the Spearman's rank correlation coefficient $(\rho)$. Statistical analyses were performed using IBM SPSS Statistics 21 software (Release version 21.0.0.0, IBM Corp., Armonk, NY, USA).

\section{References}

1. Kim, Y. et al. Temporal trends in population-based death rates associated with chronic liver disease and liver cancer in the United States over the last 30 years. Cancer 120, 3058-3065 (2014).

2. Bataller, R. \& Brenner, D. A. Liver fibrosis. J. Clin. Invest. 115, 209-218 (2005).

3. Kim, V. N. MicroRNA biogenesis: coordinated cropping and dicing. Nat. Rev. Mol. Cell Biol. 6, 376-385 (2005).

4. Bartel, D. P. MicroRNAs: target recognition and regulatory functions. Cell 136, 215-233 (2009).

5. Bartel, D. P. MicroRNAs: genomics, biogenesis, mechanism, and function. Cell 116, 281-297 (2004).

6. MacFarlane, L.-A. \& Murphy, P. R. MicroRNA: biogenesis, function and role in cancer. Curr. Genomics 11, 537-561 (2010).

7. Choi, S. S., Omenetti, A., Syn, W.-K. \& Diehl, A. M. The role of Hedgehog signaling in fibrogenic liver repair. Int. J. Biochem. Cell Biol. 43, 238-244 (2011).

8. Omenetti, A., Choi, S., Michelotti, G. \& Diehl, A. M. Hedgehog signaling in the liver. J. Hepatol. 54, 366-373 (2011).

9. Jung, Y. et al. Signals from dying hepatocytes trigger growth of liver progenitors. Gut 59, 655-665 (2010).

10. Rangwala, F. et al. Increased production of sonic hedgehog by ballooned hepatocytes. J. Pathol. 224, 401-410 (2011).

11. Chen, Y. et al. Hedgehog controls hepatic stellate cell fate by regulating metabolism. Gastroenterology 143, 1319-1329.e1311 (2012).

12. Yang, L. et al. Sonic hedgehog is an autocrine viability factor for myofibroblastic hepatic stellate cells. J. Hepatol. 48, 98-106 (2008).

13. Shah, P. N., Lopina, S. T. \& Yun, Y. H. Blends of novel L-tyrosine based polyurethanes and polyphosphate for potential biomedical applications. J. Appl. Polym. Sci. 114, 3235-3247 (2009).

14. Shah, P. N. \& Yun, Y. H. Cellular interactions with biodegradable polyurethanes formulated from L-tyrosine. J. Biomater. Appl. 27, 1017-1031 (2013). 
15. Shi, J., Aisaki, K., Ikawa, Y. \& Wake, K. Evidence of hepatocyte apoptosis in rat liver after the administration of carbon tetrachloride. Am. J. Pathol. 153, 515-525 (1998).

16. Yin, C., Evason, K. J., Asahina, K. \& Stainier, D. Y. Hepatic stellate cells in liver development, regeneration, and cancer. J. Clin. Invest. 123, 1902-1910 (2013).

17. Wunsch, E. et al. Liver expression of sulphotransferase 2A1 enzyme is impaired in patients with primary sclerosing cholangitis: lack of the response to enhanced expression of PXR. J. Immunol. Res. 2015, 1-8 (2015).

18. Bataller, R. \& Brenner, D. A. Hepatic stellate cells as a target for the treatment of liver fibrosis. Semin. Liver Dis. 21, 437-452 (2001).

19. Reeves, H. L. \& Friedman, S. L. Activation of hepatic stellate cells-a key issue in liver fibrosis. Front. Biosci. 7, 808-826 (2002)

20. Choi, S. S. et al. Hedgehog pathway activation and epithelial-to-mesenchymal transitions during myofibroblastic transformation of rat hepatic cells in culture and cirrhosis. Am. J. Physiol. Gastrointest. Liver Physiol. 297, G1093-G1106 (2009).

21. Friedman, S. L., Roll, F. J., Boyles, J. \& Bissell, D. M. Hepatic lipocytes: the principal collagen-producing cells of normal rat liver. Proc. Natl Acad. Sci. USA 82, 8681-8685 (1985).

22. Betel, D., Wilson, M., Gabow, A., Marks, D. S. \& Sander, C. The microRNA. org resource: targets and expression. Nucleic Acids Res. 36, D149-D153 (2008).

23. Pritchett, J. et al. Osteopontin is a novel downstream target of SOX9 with diagnostic implications for progression of liver fibrosis in humans. Hepatology 56, 1108-1116 (2012).

24. Kisseleva, T. et al. Myofibroblasts revert to an inactive phenotype during regression of liver fibrosis. Proc. Natl. Acad. Sci. USA 109, 9448-9453 (2012).

25. Friedman, S. L. Hepatic stellate cells: protean, multifunctional, and enigmatic cells of the liver. Physiol. Rev. 88, 125-172 (2008).

26. Schmittgen, T. D. et al. Real-time PCR quantification of precursor and mature microRNA. Methods 44, 31-38 (2008).

27. Wang, S. et al. Potential role of Hedgehog pathway in liver response to radiation. PLoS ONE 8, e74141 (2013).

28. Bellafante, E. et al. Hepatic-specific activation of peroxisome proliferator-activated receptor $\gamma$ coactivator- $1 \beta$ protects against steatohepatitis. Hepatology 57, 1343-1356 (2013).

29. Nagalingam, R. S. et al. Deficiency of cardiomyocyte-specific microRNA-378 contributes to the development of cardiac fibrosis involving a transforming growth factor $\beta$ (TGF $\beta 1$ )-dependent paracrine mechanism. J. Biol. Chem. 289, 27199-27214 (2014)

30. Suzuki, Y. \& Nakayama, M. Differential profiles of genes expressed in neonatal brain of 129X1/SvJ and C57BL/6J mice: A database to aid in analyzing DNA microarrays using nonisogenic gene-targeted mice. DNA Res. 10, 263-275 (2003).

31. Fan, X., Shao, L., Fang, H., Tong, W. \& Cheng, Y. Cross-platform comparison of microarray-based multiple-class prediction. PLoS ONE 6, e16067 (2011).

32. Li, W. Q. et al. The rno-miR-34 family is upregulated and targets ACSL1 in dimethylnitrosamine-induced hepatic fibrosis in rats. FEBS J. 278, 1522-1532 (2011).

33. Ameres, S. L. \& Zamore, P. D. Diversifying microRNA sequence and function. Nat. Rev. Mol. Cell Biol. 14, 475-488 (2013).

34. Friedman, S. L. et al. Isolated hepatic lipocytes and kupffer cells from normal human liver: morphological and functional characteristics in primary culture. Hepatology 15, 234-243 (1992).

35. Sicklick, J. K. et al. Role for hedgehog signaling in hepatic stellate cell activation and viability. Lab. Invest. 85, 1368-1380 (2005).

36. Xie, G. et al. Hedgehog signalling regulates liver sinusoidal endothelial cell capillarisation. Gut 62, 299-309 (2013).

37. de Almeida Pereira, T. et al. Viral factors induce Hedgehog pathway activation in humans with viral hepatitis, cirrhosis, and hepatocellular carcinoma. Lab. Invest. 90, 1690-1703 (2010).

38. Witek, R. P. et al. Liver cell-derived microparticles activate hedgehog signaling and alter gene expression in hepatic endothelial cells. Gastroenterology 136, 320-330.e322 (2009)

39. Buttitta, L., Mo, R., Hui, C.-C. \& Fan, C.-M. Interplays of Gli2 and Gli3 and their requirement in mediating Shh-dependent sclerotome induction. Development 130, 6233-6243 (2003).

40. $\mathrm{Hu}, \mathrm{M}$. C. et al. GLI3-dependent transcriptional repression of Gli1, Gli2 and kidney patterning genes disrupts renal morphogenesis. Development 133, 569-578 (2006)

41. Hyun, J., Wang, S., Kim, J., Kim, G. J. \& Jung, Y. MicroRNA125b-mediated Hedgehog signaling influences liver regeneration by chorionic plate-derived mesenchymal stem cells. Sci. Rep. 5, 14135 (2015).

42. Oeckinghaus, A. \& Ghosh, S. The NF- $\kappa$ B family of transcription factors and its regulation. Cold Spring Harb. Perspect. Biol. 1, a000034 (2009).

43. Ghosh, S., May, M. J. \& Kopp, E. B. NF-кB and Rel proteins: evolutionarily conserved mediators of immune responses. Annu. Rev. Immunol. 16, 225-260 (1998)
44. Roderburg, C. et al. Micro-RNA profiling reveals a role for miR-29 in human and murine liver fibrosis. Hepatology 53, 209-218 (2011).

45. Ma, X., Buscaglia, L. E. B., Barker, J. R. \& Li, Y. MicroRNAs in NF- $\kappa$ B signaling. J. Mol. Cell Biol. 3, 159-166 (2011).

46. Bruix, J., Boix, L., Sala, M. \& Llovet, J. M. Focus on hepatocellular carcinoma Cancer Cell 5, 215-219 (2004).

47. Ascha, M. S. et al. The incidence and risk factors of hepatocellular carcinoma in patients with nonalcoholic steatohepatitis. Hepatology 51, 1972-1978 (2010).

48. Chan, I. S. et al. Paracrine Hedgehog signaling drives metabolic changes in hepatocellular carcinoma. Cancer Res. 72, 6344-6350 (2012).

49. Huang, S. et al. Activation of the hedgehog pathway in human hepatocellular carcinomas. Carcinogenesis 27, 1334-1340 (2006).

50. Zhao, W. et al. Activated hepatic stellate cells promote hepatocellular carcinoma development in immunocompetent mice. Int. J. Cancer 129, 2651-2661 (2011).

51. Amann, T. et al. Activated hepatic stellate cells promote tumorigenicity of hepatocellular carcinoma. Cancer Sci. 100, 646-653 (2009).

52. Carloni, V., Luong, T. V. \& Rombouts, K. Hepatic stellate cells and extracellular matrix in hepatocellular carcinoma: more complicated than ever. Liver Int. 34, 834-843 (2014)

53. Li, L., Gao, Q., Wang, X. \& Guo, Z. [miR-378 suppresses HBV-related hepatocellular carcinoma tumor growth by directly targeting the insulin-like growth factor 1 receptor]. Zhonghua Gan Zang Bing Za Zhi 21, 609-613 (2013).

54. An, J. et al. A genetic variant in primary miR-378 is associated with risk and prognosis of hepatocellular carcinoma in a Chinese population. PLoS ONE 9, e93707 (2014).

55. Tu, X. et al. MicroRNA-101 suppresses liver fibrosis by targeting TGF $\beta$ signaling pathway. J. Pathol. 234, 46-59 (2014).

56. Su, S. et al. miR-142-5p and miR-130a-3p are regulated by IL-4 and IL-13 and control profibrogenic macrophage program. Nat. Commun. 6, 8523 (2015).

57. Ditto, A. J., Shah, P. N., Gump, L. R. \& Yun, Y. H. Nanospheres formulated from L-tyrosine polyphosphate exhibiting sustained release of polyplexes and in vitro controlled transfection properties. Mol. Pharm. 6, 986-995 (2009)

58. Ditto, A. J. et al. In vivo gene delivery with l-tyrosine polyphosphate nanoparticles. Mol. Pharm. 10, 1836-1844 (2013).

59. Yang, B. B., Lum, P. K., Hayashi, M. M. \& Roskos, L. K. Polyethylene glycol modification of filgrastim results in decreased renal clearance of the protein in rats. J. Pharm. Sci. 93, 1367-1373 (2004)

60. Yi, H.-S. et al. Treatment with 4-methylpyrazole modulated stellate cells and natural killer cells and ameliorated liver fibrosis in mice. PLOS ONE 10, e0127946 (2015)

61. Suh, Y. G. et al. CD11b + Gr1 + bone marrow cells ameliorate liver fibrosis by producing interleukin-10 in mice. Hepatology 56, 1902-1912 (2012).

62. Philips, G. M. et al. Hedgehog signaling antagonist promotes regression of both liver fibrosis and hepatocellular carcinoma in a murine model of primary liver cancer. PLoS ONE 6, e23943 (2011).

63. Horiguchi, N. et al. Cell type-dependent pro-and anti-inflammatory role of signal transducer and activator of transcription 3 in alcoholic liver injury. Gastroenterology 134, 1148-1158 (2008).

64. Radaeva, S. et al. Natural killer cells ameliorate liver fibrosis by killing activated stellate cells in nkg2d-dependent and tumor necrosis factor-related apoptosisinducing ligand-dependent manners. Gastroenterology 130, 435-452 (2006).

65. Sun, R., Jaruga, B., Kulkarni, S., Sun, H. \& Gao, B. IL-6 modulates hepatocyte proliferation via induction of $\mathrm{HGF} / \mathrm{p} 21^{\mathrm{cip} 1}$ : Regulation by SOCS3. Biochem. Biophys. Res. Commun. 338, 1943-1949 (2005).

66. Smedsrød, B. \& Pertoft, H. Preparation of pure hepatocytes and reticuloendothelial cells in high yield from a single rat liver by means of Percoll centrifugation and selective adherence. J. Leukoc. Biol. 38, 213-230 (1985).

67. Jeong, D. et al. MicroRNA-124 links p53 to the NF- $\kappa$ B pathway in B-cell lymphomas. Leukemia 29, 1868-1874 (2015).

68. Ozpolat, B., Sood, A. \& Lopez-Berestein, G. Nanomedicine based approaches for the delivery of siRNA in cancer. J. Intern. Med. 267, 44-53 (2010).

\section{Acknowledgements}

The biospecimens for this study were provided by the Pusan National University Hospital, a member of the National Biobank of Korea, which is supported by the Ministry of Health and Welfare. This work was supported by the National Research Foundation (NRF) of Korea funded by the Korean Government (MEST) (2013R1A2A2A01068268) to Y.J. and the National Science Foundation (USA, CAREER Award, CBET-0954360) to YY.

\section{Author Contributions}

J.H., Y.Y. and Y.J. conceived and designed the study. J.H., S.W., K.R., S.P. and J.K. carried out experiments. J.H. analysed the data. J.H., S.W.K., Y.Y. and Y.J. interpreted the data 
I.C., C.H. and Y.J. contributed reagents/materials/analysis tools. J.H. and Y.J. wrote the manuscript. Y.J. and Y.Y. obtained funding. All authors critically revised the manuscript and approved its final version.

\section{Additional information}

Accession codes: The microarray data have been deposited in NCBI GEO database under the accession code GSE77271.

Supplementary Information accompanies this paper at http://www.nature.com/ naturecommunications

Competing financial interests: The authors declare no competing financial interests.
Reprints and permission information is available online at http://npg.nature.com/ reprintsandpermissions/

How to cite this article: Hyun, J. et al. MicroRNA-378 limits activation of hepatic stellate cells and liver fibrosis by suppressing Gli3 expression. Nat. Commun. 7:10993 doi: 10.1038/ncomms10993 (2016).

(c) (i) This work is licensed under a Creative Commons Attribution 4.0 International License. The images or other third party material in this article are included in the article's Creative Commons license, unless indicated otherwise in the credit line; if the material is not included under the Creative Commons license, users will need to obtain permission from the license holder to reproduce the material. To view a copy of this license, visit http://creativecommons.org/licenses/by/4.0/ 